\title{
Hepatic transcriptome analysis of inter-family variability in flesh $n-3$ long-chain polyunsaturated fatty acid content in Atlantic salmon
}

\author{
Sofia Morais ${ }^{1 *}$, John B Taggart ${ }^{1}$, Derrick R Guy², J Gordon Bell ${ }^{1}$ and Douglas R Tocher ${ }^{1}$
}

\begin{abstract}
Background: Genetic selection of Atlantic salmon families better adapted to alternative feed formulations containing high levels of vegetable ingredients has been suggested to ensure sustainable growth of aquaculture. The present study aimed to identify molecular pathways that could underlie phenotypic differences in flesh n-3 long-chain polyunsaturated fatty acid (LC-PUFA) levels when fish are fed vegetable oil diets. Liver transcriptome was analyzed and compared in four families presenting higher or lower n-3 LC-PUFA contents at two contrasting flesh total lipid levels.
\end{abstract}

Results: The main effect of n-3 LC-PUFA contents was in the expression of immune response genes (38\% of all significantly affected genes), broadly implicated in the modulation of inflammatory processes and innate immune response. Although genetic evaluations of traits used in the breeding program revealed that the chosen families were not balanced for viral disease resistance, this did not fully explain the preponderance of immune response genes in the transcriptomic analysis. Employing stringent statistical analysis no lipid metabolism genes were detected as being significantly altered in liver when comparing families with high and low n-3 LC-PUFA flesh contents. However, relaxing the statistical analysis enabled identification of potentially relevant effects, further studied by RT-qPCR, in cholesterol biosynthesis, lipoprotein metabolism and lipid transport, as well as eicosanoid metabolism particularly affecting the lipoxygenase pathway. Total lipid level in flesh also showed an important effect on immune response and $8 \%$ of significantly affected genes related to lipid metabolism, including a fatty acyl elongase (elov/2), an acyl carrier protein and stearoyl-CoA desaturase.

Conclusions: Inter-family differences in n-3 LC-PUFA content could not be related to effects on lipid metabolism, including transcriptional modulation of the LC-PUFA biosynthesis pathway. An association was found between flesh adiposity and n-3 LC-PUFA in regulation of cholesterol biosynthesis, which was most likely explained by variation in tissue n-3 LC-PUFA levels regulating transcription of cholesterol metabolism genes through srebp2. A preponderance of immune response genes significantly affected by n-3 LC-PUFA contents could be potentially associated with disease resistance, possibly involving anti-inflammatory actions of tissue n-3 LC-PUFA through eicosanoid metabolism. This association may have been fortuitous, but it is important to clarify if this trait is included in future salmon breeding programmes.

\footnotetext{
* Correspondence: sofia.morais@irta.cat

'Institute of Aquaculture, University of Stirling, Stirling, FK9 4LA Scotland, UK

Full list of author information is available at the end of the article
} 


\section{Background}

Aquaculture is the fastest growing animal production activity worldwide, supplying an increasing proportion of fish for human consumption, estimated at around $50 \%$ of total supply in 2008 [1]. However, the growth of marine aquaculture is threatened by its excessive reliance on fishmeal (FM) and fish oil (FO) from wild stocks for the production of fish feeds, which is also an ecologically unsound practice. Almost $89 \%$ of the total global production of FO is currently used by aquaculture [2] and the future of this activity strongly depends on the reduction of dependency on FM and FO and its replacement with alternative ingredients, such as vegetable oils (VO) and plant meals, while maintaining fish welfare and health benefits for the human consumer. Fish are highly nutritious components of the human diet and the main source of essential n-3 long-chain polyunsaturated fatty acids (LC-PUFA). The beneficial effects of fatty acids, such as eicosapentaenoic acid (EPA) and docosahexaenoic acid (DHA), are numerous and important, including protection against a range of cardiovascular and inflammatory diseases, as well as neurological disorders [3]. Atlantic salmon (Salmo salar) can grow well on diets where FO has been completely replaced by VO but this results in lower levels of n-3 LC-PUFA in their flesh, compromising their nutritional value and health-promoting effects to the human consumer [4].

The use of selective breeding programs to enhance traits of commercial importance is becoming increasingly common in aquaculture [5]. It has been suggested that combining genetic selection for fish that are more efficient in retaining and/or biosynthesising n-3 LCPUFA with changes in commercial diet formulations (i.e., higher levels of inclusion of VO) might be a viable strategy to meet growing worldwide demands for aquaculture products, without loss of nutritional value. Previous studies have shown wide individual variability in the capacity of Atlantic salmon to retain or synthesize n-3 LC-PUFA when fed VO diets [6]. Following this, Leaver et al. [7] demonstrated that deposition and/or retention in flesh of dietary n-3 LC-PUFA, EPA and DHA, is a highly heritable trait $\left(h^{2}=0.77\right)$ in salmon. These results have prompted further interest in large-scale in-depth studies exploring genotype $\times$ nutrient interactions in salmon, analysing whether the genetic background of the fish could affect the physiological response to complete dietary replacement of FO by VO $[8,9]$. In the present study we investigated this further by analyzing the transcriptome from liver, the primary site of synthesis and export of lipids to extra-hepatic tissues including flesh, from four Atlantic salmon families phenotyped for different levels of flesh n-3 LC-PUFA content in response to a VO diet. The objective was to identify gene pathways and molecular mechanisms that might underlie differences in flesh n-3 LC-PUFA contents when salmon families were fed the same low LC-PUFA diet. Furthermore, because n-3 LC-PUFA level is a component of, and associated with total lipid content in a tissue, a factorial design was chosen in which families containing higher and lower proportions of flesh n-3 LC-PUFA were compared at similar flesh total lipid contents.

\section{Results}

\section{Family lipid contrasts}

Lipid analysis of fifty Atlantic salmon families showed flesh lipid levels ranging from 2.3 to $5.7 \%$ of wet weight, with relative and absolute n-3 LC-PUFA contents varying from 71 to $136(\mu \mathrm{g} / \mathrm{mg}$ lipid) and 314 to $554(\mathrm{mg} /$ $100 \mathrm{~g}$ flesh), respectively. As expected, high correlations between lipid level and n-3 LC-PUFA content were observed ( $\mathrm{r}=-0.65$ or 0.70 for relative and absolute contents, respectively), indicating that only families with near identical lipid levels should be compared to avoid confounding effects associated with the lipid level factor (additional file 1). Using these results, four families were identified; two with high $(\mathrm{H})$ levels of lipid (5 g/100 g flesh), and two with low (L) levels of lipid (3.5 g/100 g flesh) and, within each level of total lipid, the two families had significantly contrasting relative n-3 LC-PUFA contents (similarly termed $\mathrm{H}$ and $\mathrm{L}$ ). Therefore, the four families constituted a 2 × 2 factorial design, labelling each family by the total lipid/n-3 LC-PUFA contrasts as LL, LH, HL and $\mathrm{HH}$, respectively (Table 1), which allowed comparisons of n-3 LC-PUFA contents at a constant lipid level and, similarly, comparisons of total lipid at constant n-3 LC-PUFA levels.

\section{Microarray analysis}

A two-way ANOVA analysis employing the BenjaminiHochberg multiple testing correction (at a significance level of 0.05 and fold change cut-off of 1.2) was performed to assess significant effects of the factors ' $n-3$ LC-PUFA' and 'total lipid', which returned lists with 43, 109 and 66 entities for each factor and their interaction, respectively. These significant lists were then analyzed in detail and genes were categorized according to their biological function, in some cases inferred from mammalian homolog genes (Tables 2 and 3). Because the focus of this work was to identify genes that are specifically affected by the trait n-3 LC-PUFA content without the interference of total lipid level, the interaction between the two factors is not presented. Distribution of genes by categories of biological function (excluding $12-18 \%$ non-annotated probes, those representing the same gene or with a miscellaneous function) revealed that there was a preponderance of immune response genes significantly affected by both factors: $38 \%$ by ' $n-3$ LC-PUFA' and 29\% by 'total lipid'. Gene Ontology (GO) 
Table 1 Lipid phenotypes of families chosen for molecular analysis

\begin{tabular}{|c|c|c|c|c|c|}
\hline \multirow[t]{2}{*}{ Family } & \multirow{2}{*}{$\begin{array}{c}\text { Total Lipid } \\
\text { (g/100 g flesh) }\end{array}$} & \multicolumn{2}{|c|}{ n-3 LC-PUFA } & \multicolumn{2}{|c|}{ ARA } \\
\hline & & $\begin{array}{c}\text { Relative } \\
(\mu \mathrm{g} / \mathrm{mg} \text { lipid })\end{array}$ & $\begin{array}{c}\text { Absolute } \\
\text { (mg/100 } \mathrm{g} \text { flesh) }\end{array}$ & $\begin{array}{c}\text { Relative } \\
(\mu \mathrm{g} / \mathrm{mg} \text { lipid) }\end{array}$ & $\begin{array}{c}\text { Absolute } \\
\text { (mg/100 g flesh) }\end{array}$ \\
\hline LL & $3.5 \pm 0.4$ & $105.1 \pm 3.8 *$ & $363.0 \pm 30.3$ & $3.45 \pm 0.13 *$ & $11.93 \pm 1.00$ \\
\hline LH & $3.5 \pm 0.7$ & $133.8 \pm 4.8 *$ & $468.0 \pm 92.9$ & $4.25 \pm 0.06$ * & $14.87 \pm 2.94$ \\
\hline $\mathrm{HL}$ & $5.1 \pm 0.8$ & $83.7 \pm 14.0 *$ & $426.9 \pm 103.2$ & $2.70 \pm 0.53$ & $13.81 \pm 3.80$ \\
\hline $\mathrm{HH}$ & $5.0 \pm 0.7$ & $112.0 \pm 7.9 *$ & $554.3 \pm 50.7$ & $3.67 \pm 0.63$ & $18.04 \pm 2.09$ \\
\hline
\end{tabular}

Indicated are levels of total lipid (g/100 g flesh, wet weight), and relative and absolute contents of total n-3 LC-PUFA and of the $\mathrm{n}-6$ LC-PUFA arachidonic acid (ARA) in the flesh ( $n=3$ pools) of the 4 Atlantic salmon families used in the transcriptomic analysis. Asterisks signify significant differences between the two families with the same total lipid content.

enrichment analysis, which enables the identification of GO terms significantly enriched in the input entity list when compared to the whole array dataset, revealed that this is a true over-representation in the list of genes significantly affected by the 'total lipid' factor (Additional file 2). In contrast, genes involved in the broad category of metabolism only corresponded to $21 \%$ of genes significantly affected by n-3 LC-PUFA content and 30\% by the 'total lipid' factor. Surprisingly, no lipid metabolism genes were significantly altered in liver when comparing families with higher and lower contents of n-3 LC-PUFA in their flesh, while about $8 \%$ were significantly affected by flesh lipid level. Within these, noteworthy was the down-regulation of fatty acyl elongase (elovl2) and of acyl carrier protein transcripts in salmon having a higher lipid level in their flesh, independent of LC-PUFA content. On the other hand, stearoyl-CoA desaturase was significantly up-regulated in fish with higher lipid levels in their flesh. The interaction between both factors is not presented but it did not substantially affect lipid metabolism genes. Finally, and in general, genes involved in regulation of transcription and signalling were also prevalent, $17 \%$ in response to 'n-3 LC-PUFA' and 12$13 \%$ to 'total lipid'.

Therefore, the results did not identify lipid metabolism pathways that might underlie differences in flesh n-3 LCPUFA composition between families. However, previous studies demonstrated that hepatic metabolism genes typically show only low fold changes, even when comparing highly contrasting nutritional compositions (e.g., inclusion of $100 \%$ FO versus $100 \% \mathrm{VO}$ in diets $[8,9]$ ), compared to immune response genes that tend to be regulated with higher magnitudes of change [10]. Hence, nutritional data such as the present data have been analysed previously without multiple testing correction and this was found to result in relevant biological interpretations, when validated by reverse transcription real time quantitative PCR (RT-qPCR) [9,11]. For this reason, we examined the significant effects of 'n-3 LC-PUFA' without the correction, and from within the list containing 1951 features (significance level, 0.05; fold change cut-off, 1.2), we identified and categorized all 48 lipid metabolism transcripts present (Table 4). An effect on cholesterol metabolism was apparent for the factor ' $n-3$ LC-PUFA', with several genes of the biosynthesis pathway and its regulation being down-regulated in fish with a high n-3 LC-PUFA phenotype. In addition, glycerophospholipid synthesis, lipid hydrolysis and eicosanoid synthesis and metabolism were also affected, while other genes were associated with lipid and fatty acid transport, fatty acid synthesis and regulation of lipid metabolism.

\section{Validation of results by RT-qPCR}

To validate the microarray analysis results, expression of selected genes was quantified by RT-qPCR. These genes were chosen from lipid metabolism pathways that were more highly affected by the factor ' $n$-3 LC-PUFA', and also included immune response genes, which was the category most highly affected by both 'n-3 LC-PUFA' and 'total lipid' factors. In addition, the expression of two fatty acyl desaturases ( $\triangle 5 \mathrm{fad}$ and $\Delta 6 \mathrm{fad})$ and one elongase (elovl2), which are typically responsive to dietary levels of $n-3$ LC-PUFA were also determined. The LC-PUFA biosynthesis pathway was not identified by the microarray analysis as being differentially expressed in families with different n-3 LC-PUFA flesh contents but, given the potential importance of this pathway in determining n-3 PUFA phenotypes, we specifically aimed to verify this result. The RT-qPCR results confirmed that genes involved in LC-PUFA biosynthesis were not differentially expressed in families with higher and lower levels of n-3 LC-PUFA (Table 5). Furthermore, the RT-qPCR results confirmed significant downregulation of genes involved in hepatic cholesterol biosynthesis, such as isopentenyl-diphosphate isomerase (ipi), 7-dehydrocholesterol reductase (7dchr) and sterol regulatory element-binding protein $2(s r e b p 2)$ in families containing higher levels of n-3 LC-PUFA in their flesh although this was only observed when this phenotype was also associated with low lipid level, except for $7 d c h r$, which was significantly down-regulated irrespective of lipid level. With regards to lipoprotein metabolism (lipid transport) genes, general trends such as the magnitude and direction of change were broadly similar between 
Table 2 Liver transcripts differentially expressed when examining the explanatory power of the factor ' $n-3$ LC-PUFA' content in flesh of four families of Atlantic salmon fed the same low FM/high VO diet

\begin{tabular}{lll}
\hline Probe name & Gene & High/Low LC-PUFA \\
\cline { 2 - 3 } & & L Lipid (LH/LL) \\
\hline Metabolism (21\%)
\end{tabular}

Metabolism (21\%)

Energy metabolism (4\%)

Ssa\#\$31995754 Cytochrome c oxidase subunit 2

$\begin{array}{rrr}7.1 & -1.0 & 0.0001 \\ 3.7 & 79.8 & 0.0011 \\ -14.9 & -1.1 & 0.0026 \\ -3.3 & -1.4 & 0.0188\end{array}$

Protein and amino acid metabolism (13\%)

$\begin{array}{llr}\text { Ssa\#CB502423 } & \text { N-acetylated alpha-linked acidic dipeptidase-like } 1 & 3.7 \\ \text { Ssa\#STIR03710 } & \text { Proteasome subunit beta type-9 precursor } & -14.9 \\ \text { Ssa\#S31993738_S } & \text { Ubiquitin-conjugating enzyme E2 } & -3.3 \\ \text { Xenobiotic and oxidant metabolism (4\%) } & \end{array}$

$\begin{array}{ll}\text { Ssa\#S18892279 } & \text { Cytochrome P450 1A } \\ \text { Ssa\#STIR00161_2 } & \text { Cytochrome P450 1A } \\ \text { Ssa\#STIR00161_3 } & \text { Cytochrome P450 1A } \\ \text { Con_CANDS_13 } & \text { Cytochrome P450 1A }\end{array}$

Con_CANDS_13

Cytochrome P450 1A

Translation (8\%)

Ssa\#STIR26031 Mitochondrial 28 S ribosomal protein S34

Ssa\#S18867312

Ribonuclease UK114

Regulation of transcription (17\%)

\begin{tabular}{|c|c|}
\hline Ssa\#S35510106 & Zinc finger protein 367 \\
\hline Omy\#S18104058 & Zinc finger protein 235 \\
\hline Ssa\#TC111702 & Reverse transcriptase-like protein \\
\hline Ssa\#TC112002 & Retinoid $X$ receptor beta \\
\hline \multicolumn{2}{|c|}{ Signalling and protein modification (17\%) } \\
\hline Ssa\#STIR15776 & $\begin{array}{l}\text { Dolichyl-diphosphooligosaccharide-protein } \\
\text { glycosyltransferase subunit } 4\end{array}$ \\
\hline Ssa\#STIR23530 & $\begin{array}{l}\text { Dolichyl-diphosphooligosaccharide-protein } \\
\text { glycosyltransferase subunit } 4\end{array}$ \\
\hline Ssa\#STIR03642 & $\begin{array}{l}\text { Dolichyl-diphosphooligosaccharide-protein } \\
\text { glycosyltransferase subunit } 4\end{array}$ \\
\hline Ssa\#STIR01857 & $\begin{array}{l}\text { Dolichyl-diphosphooligosaccharide-protein } \\
\text { glycosyltransferase subunit } 4\end{array}$ \\
\hline Ssa\#STIR31840 & $\begin{array}{l}\text { Sphingomyelin phosphodiesterase acid transcript } \\
\text { variant } 1\end{array}$ \\
\hline Ssa\#STIR07369 & RAF1 proto-oncogene serine/threonine-protein kinas \\
\hline Ssa\#S35552908 & $\begin{array}{l}\text { Tyrosine 3-monooxygenase/tryptophan } \\
\text { 5-monooxygenase activation protein, epsilon polyper }\end{array}$ \\
\hline \multicolumn{2}{|c|}{ Immune response (38\%) } \\
\hline Ssa\#S35536179 & similar to novel NACHT domain containing protein \\
\hline Ssa\#S35516341 & Tripartite motif-containing protein 25 (trim25) \\
\hline Ssa\#S30241035 & MHC class I \\
\hline Ssa\#STIR02298 & c-c motif chemokine 13 precursor (ccl13) \\
\hline Ssa\#S35581943 & Myelin and lymphocyte protein (mal) \\
\hline Ssa\#KSS3969 & Leukocyte cell-derived chemotaxin 2 precursor (lect2) \\
\hline Ssa\#STIR15577 & Tissue factor pathway inhibitor a \\
\hline Omy\#S15332652 & Pentraxin \\
\hline Ssa\#TC70262 & Cathepsin K \\
\hline Ssa\#S35558945 & Tripartite motif-containing protein 25 (trim25) \\
\hline
\end{tabular}

0.0096

0.0160

0.0213

0.0494

0.0017

0.0450

0.0026

0.0058

0.0104

0.0134

- 19.1

123.3

0.0000

0.0000

0.0000

0.0001

0.0303

0.0343

0.0455

$-2.1$

0.0026

0.0066

0.0079

0.0100

0.0100

0.0134

0.0135

0.0172

0.0279

0.0347 
Table 2 Liver transcripts differentially expressed when examining the explanatory power of the factor ' $n-3$ LC-PUFA' content in flesh of four families of Atlantic salmon fed the same low FM/high VO diet (Continued)

\begin{tabular}{|c|c|c|c|c|}
\hline \multicolumn{5}{|c|}{ Miscellaneous/unknown function } \\
\hline Ssa\#STIR09736 & Transmembrane protein 42 & 2.3 & 1.9 & 0.0009 \\
\hline Ssa\#S35519407 & Tetratricopeptide repeat protein 23 & 3.7 & 1.0 & 0.0011 \\
\hline Ssa\#STIR02307 & $\begin{array}{l}\text { Family with sequence similarity member } \\
\text { a (FAM36A) }\end{array}$ & 1.3 & 3.6 & 0.0026 \\
\hline Omy\#BX309274 & $\begin{array}{l}\text { X-ray repair complementing defective repair in } \\
\text { Chinese hamster cells } 3\end{array}$ & -1.0 & -8.0 & 0.0100 \\
\hline Ssa\#STIR21287 & Solute carrier family 30 (zinc transporter) member 7 & 2.1 & 1.5 & 0.0100 \\
\hline Ssa\#S35521859_S & Family with sequence similarity member a (FAM36A) & -1.1 & 4.8 & 0.0358 \\
\hline Ssa\#S18842295 & Alveolin & 1.7 & 4.6 & 0.0422 \\
\hline
\end{tabular}

Results were obtained by two-way ANOVA analysis ( $\mathrm{p}<0.05$; fold change cut-off of 1.2) with Benjamini-Hochberg multiple testing correction. Transcripts (43 probes; $12 \%$ unknowns) are arranged by categories of biological function and, within these, by p-value. Indicated are also the probe names and the expression ratio determined separately between families with high vs low n-3 LC-PUFA contents, for each total lipid level. Percentages of distribution of genes within each category do not include non-annotated probes, those representing the same gene or with a miscellaneous function.

the microarray and the RT-qPCR analysis for the high versus low n-3 LC-PUFA comparison at low lipid contents, although RT-qPCR results were not significant. In the case of high lipid contents, the match between microarray and RT-qPCR data was less consistent, except for lipoprotein lipase $(l p l)$, which was similarly up-regulated albeit nonsignificantly. Up-regulation of the glycerophospholipid biosynthesis pathway in fish with higher n-3 LC-PUFA contents was also indicated when associated with high lipid levels, significant for monoacylglycerol Oacyltransferase 1 (mgat). With regards to the eicosanoid biosynthesis pathway, the microarray results could only be confirmed for arachidonic 5-lipoxygenase (alox5). Validation of lipid metabolism genes affected by the 'total lipid' factor (Table 6) confirmed the lower expression of elovl2 in salmon presenting higher lipid levels in their flesh, independent of LC-PUFA content. Finally, good agreement was found between the microarray and RT-qPCR results for immune response genes in response to both 'n-3 LC-PUFA' (Table 5) and 'total lipid' (Table 6) factors.

\section{Genetic evaluations}

Subsequent to the dietary trial and microarray analyses, genetic evaluations (estimated breeding values, EBVs) became available for a range of traits upon which the families are under active selection in the breeding program. Given the unexpectedly high preponderance of immune response genes identified by transcriptomic analysis, we investigated associations with traits that could potentially explain the gene expression data. In this respect, one of the most relevant traits was 'survival to infectious pancreatic necrosis (IPN) virus', known to be almost entirely controlled by a major QTL [12]. Genetic evaluations included data collected from a freshwater experimental IPN challenge on full-sibs from the same families as the trial fish. Examining the families, selected on their lipid phenotypes, used for transcriptomic analysis it was seen that family $\mathrm{HH}$, containing both high total lipid and high n-3 LC-PUFA flesh contents, also showed a high EBV for survival to IPN (selection differential on a standardized normal distribution $=1.86$ standard deviations), contrasting with -0.83 (LL) -0.99 (LH) and -1.28 (HL) for the other families, that could introduce a potential for bias in interpretation of the transcriptomic responses. However, no such imbalance was present in the lower lipid grouping, comparing families LL and LH (additional file 3).

\section{Discussion}

The present study which ascertained lipid profiles of 50 Atlantic salmon families confirmed previous results showing important inter-family variation in the ability to retain n-3 LC-PUFA in the flesh when fish are fed diets with low levels of these fatty acids [7]. Furthermore, even though a high correlation was found between flesh lipid levels and n-3 LC-PUFA contents, families with the same total lipid level varied significantly in n-3 LC-PUFA contents. In the present study we did not examine whether these differences have a genetic basis, as this was established previously [7], but instead aimed to identify molecular pathways whose transcriptional regulation might underlie the phenotypic differences, independent of lipid content.

\section{LC-PUFA biosynthesis}

Differences in flesh n-3 LC-PUFA content in individuals fed the same diet is likely to arise from either selective incorporation and retention of fatty acids supplied by the diet or from biosynthesis from precursors in tissues such as the liver. In the present study we performed a transcriptomic study to identify molecular mechanisms potentially underlying flesh n-3 LC-PUFA phenotypes. Expression of candidate genes of the LC-PUFA biosynthesis pathway were also quantified as there was good evidence that these genes are transcriptionally regulated 
Table 3 Liver transcripts differentially expressed when examining the explanatory power of the variable 'total lipid' level in flesh of four families of Atlantic salmon fed the same low FM/high VO diet

\begin{tabular}{|c|c|c|c|c|}
\hline \multirow[t]{2}{*}{ Probe name } & \multirow[t]{2}{*}{ Gene } & \multicolumn{2}{|c|}{ High/Low Lipid } & \multirow[t]{2}{*}{ p-value } \\
\hline & & Lo LC-PUFA (HL/LL) & Hi LC-PUFA (HH/LH) & \\
\hline \multicolumn{5}{|l|}{ Metabolism (30\%) } \\
\hline \multicolumn{5}{|c|}{ Lipid metabolism (8\%) } \\
\hline Ssa\#STIR02479 & Butyrophilin subfamily 2 member A2 precursor & 7.76 & 1.23 & 0.0001 \\
\hline Ssa\#STIR03356 & Acyl carrier protein, mitochondrial precursor & -5.17 & -1.55 & 0.0004 \\
\hline Ssa\#STIR00151_3 & Polyunsaturated fatty acid elongase (elov/2) & -1.40 & -1.87 & 0.0190 \\
\hline Ssa\#STIR00151_2 & Polyunsaturated fatty acid elongase (elov/2) & -1.28 & -1.97 & 0.0238 \\
\hline Con_CANDS_03 & Polyunsaturated fatty acid elongase (elov/2) & -1.33 & -1.82 & 0.0310 \\
\hline Ssa\#STIR24266 & Acyl carrier protein, mitochondrial precursor & -2.88 & -1.52 & 0.0312 \\
\hline Ssa\#STIR21802 & Stearoyl-CoA desaturase & 3.53 & 3.17 & 0.0324 \\
\hline Ssa\#STIR00151_4 & Polyunsaturated fatty acid elongase (elov/2) & -1.41 & -1.82 & 0.0393 \\
\hline Ssa\#\#35501441_S & Acyl carrier protein, mitochondrial precursor & -2.65 & -1.44 & 0.0457 \\
\hline Ssa\#KSS4634 & Stearoyl-CoA desaturase & 3.52 & 2.82 & 0.0486 \\
\hline \multicolumn{5}{|c|}{ Energy metabolism and generation of precursor metabolites (11\%) } \\
\hline Ssa\#S31995754 & Cytochrome c oxidase subunit 2 & 6.57 & -1.12 & 0.0002 \\
\hline Ssa\#STIR03496 & LYR motif-containing protein 5 & 1.20 & 1.48 & 0.0233 \\
\hline Ssa\#STIR03740 & 6-Phosphogluconate dehydrogenase, decarboxylating & 1.60 & 1.93 & 0.0238 \\
\hline Ssa\#TC106663 & Creatine kinase, testis isozyme & 1.76 & 1.46 & 0.0380 \\
\hline Ssa\#STIR19155 & NADH dehydrogenase iron-sulfur protein 7 & 1.21 & 2.30 & 0.0409 \\
\hline Ssa\#STIR12872 & V-type ATPase B subunit & -1.19 & -1.24 & 0.0419 \\
\hline \multicolumn{5}{|c|}{ Protein and amino acid metabolism (4\%) } \\
\hline Ssa\#STIR03710 & Proteasome subunit beta type-9 precursor & 1.94 & 25.52 & 0.0001 \\
\hline Ssa\#S30294677 & Serine protease HTRA1 precursor (Serine protease 11) & 3.42 & 2.44 & 0.0089 \\
\hline \multicolumn{5}{|c|}{ Carbohydrate metabolism (4\%) } \\
\hline Ssa\#TC106766 & Glycogenin & 8.64 & 1.67 & 0.0093 \\
\hline Ssa\#S30290426 & Serine dehydratase-like & 1.60 & 1.72 & 0.0393 \\
\hline \multicolumn{5}{|c|}{ Xenobiotic and oxidant metabolism (4\%) } \\
\hline Ssa\#S35671757 & Extracellular superoxide dismutase & 2.18 & 1.09 & 0.0061 \\
\hline Ssa\#STIR25620 & Microsomal glutathione S-transferase 1 & 2.45 & 2.72 & 0.0096 \\
\hline Omy\#S18159333 & Microsomal glutathione S-transferase 1 & 2.47 & 3.15 & 0.0233 \\
\hline \multicolumn{5}{|l|}{ Transport (2\%) } \\
\hline Ssa\#S35599996 & ATPase, $\mathrm{H}+$ transporting, lysosomal, $\mathrm{V} 1$ subunit $\mathrm{H}$ & 45.10 & 15.16 & 0.0000 \\
\hline \multicolumn{5}{|l|}{ Translation (4\%) } \\
\hline Ssa\#STIR26031 & Mitochondrial 28 S ribosomal protein S34 & 1.19 & 19.22 & 0.0000 \\
\hline Ssa\#S30241612 & $39 \mathrm{~S}$ ribosomal protein L16 & 1.30 & 1.51 & 0.0324 \\
\hline \multicolumn{5}{|c|}{ Regulation of transcription (12\%) } \\
\hline Ssa\#STIR06878 & Cytosolic iron-sulfur protein assembly 1 & -1.16 & -1.56 & 0.0106 \\
\hline Ssa\#TC112002 & Retinoid $\mathrm{X}$ receptor beta & 1.20 & -16.00 & 0.0254 \\
\hline Omy\#S15320037 & $\begin{array}{l}\text { SWI/SNF-related matrix-associated actin-dependent } \\
\text { regulator of chromatin a4 }\end{array}$ & -1.97 & -1.53 & 0.0324 \\
\hline Ssa\#CN181280 & alpha thalassaemia mental retardation X-linked protein & -3.05 & -6.90 & 0.0419 \\
\hline Ssa\#S35697153 & YLP motif containing 1 & -1.49 & -1.32 & 0.0428 \\
\hline Ssa\#S35486480 & Zinc finger protein 492 & -1.08 & -2.29 & 0.0452 \\
\hline
\end{tabular}


Table 3 Liver transcripts differentially expressed when examining the explanatory power of the variable 'total lipid' level in flesh of four families of Atlantic salmon fed the same low FM/high VO diet (Continued)

\begin{tabular}{|c|c|c|c|c|}
\hline \multicolumn{5}{|c|}{ Signalling and protein modification (13\%) } \\
\hline Ssa\#STIR15776 & $\begin{array}{l}\text { Dolichyl-diphosphooligosaccharide-protein } \\
\text { glycosyltransferase subunit } 4\end{array}$ & -84.27 & 2.44 & 0.0000 \\
\hline Ssa\#STIR23530 & $\begin{array}{l}\text { Dolichyl-diphosphooligosaccharide-protein } \\
\text { glycosyltransferase subunit } 4\end{array}$ & -83.34 & 2.60 & 0.0000 \\
\hline Ssa\#STIR03642 & $\begin{array}{l}\text { Dolichyl-diphosphooligosaccharide-protein } \\
\text { glycosyltransferase subunit } 4\end{array}$ & -49.76 & 2.34 & 0.0001 \\
\hline Ssa\#STIR01857 & $\begin{array}{l}\text { Dolichyl-diphosphooligosaccharide-protein } \\
\text { glycosyltransferase subunit } 4\end{array}$ & -74.19 & 2.51 & 0.0011 \\
\hline Ssa\#S35516167 & $14 \mathrm{kDa}$ phosphohistidine phosphatase & 1.57 & 1.34 & 0.0019 \\
\hline Ssa\#STIR1 1086 & Lunatic fringe & -5.47 & -2.00 & 0.0154 \\
\hline Ssa\#S30263209 & HtrA serine peptidase 3 & 3.71 & 2.33 & 0.0154 \\
\hline Ssa\#STIR22920 & HCLS1-associated protein X-1 & 1.73 & 2.33 & 0.0390 \\
\hline Ssa\#S35701148 & Phosphatase and actin regulator 3 & -1.52 & -65.53 & 0.0404 \\
\hline Omy\#S15290792 & Serine/threonine-protein kinase PLK2 & 1.70 & 2.03 & 0.0468 \\
\hline \multicolumn{5}{|c|}{ Immune response (29\%) } \\
\hline Ssa\#STIR00130_4 & Complement factor $\mathrm{H}$ precursor (cfh) & -1.43 & -1.67 & 0.0012 \\
\hline Ssa\#S35536179 & novel NACHT domain containing protein & -1.89 & -2.41 & 0.0013 \\
\hline Ssa\#S30241035 & MHC class I & -1.55 & -30.59 & 0.0024 \\
\hline Ssa\#S35516341 & Tripartite motif-containing protein 25 (trim25) & -5.64 & -4.34 & 0.0125 \\
\hline Ssa\#S18834140 & Complement factor $\mathrm{H}$ precursor (cfh) & -1.34 & -1.57 & 0.0131 \\
\hline Omy\#utu04b09 & Complement factor $\mathrm{H}$ precursor (cfh) & -1.56 & -1.70 & 0.0134 \\
\hline Ssa\#STIR15577 & Tissue factor pathway inhibitor a & 21.03 & 1.59 & 0.0151 \\
\hline Ssa\#S35558236 & C-type lectin domain family 16 , member A & 4.59 & 2.25 & 0.0190 \\
\hline Ssa\#STIR10409 & CD83 antigen precursor & 1.98 & 1.68 & 0.0238 \\
\hline Omy\#S15332652 & Putative pentraxin & -1.33 & 159.84 & 0.0246 \\
\hline Ssa\#S35685271 & GTPase IMAP family member 7 & -168.44 & -4.09 & 0.0263 \\
\hline Ssa\#S35551959 & Duodenase-1 & -2.94 & -2.77 & 0.0349 \\
\hline Ssa\#S35558945 & Tripartite motif-containing protein 25 (trim25) & -5.74 & -4.28 & 0.0373 \\
\hline Ssa\#S35685273 & Lactose-binding lectin $1-2$ precursor putative & 1.56 & 4.04 & 0.0380 \\
\hline Ssa\#S31977617 & Scavenger receptor cysteine-rich type 1 protein $\mathrm{m} 130$ & -1.31 & -2.05 & 0.0404 \\
\hline Ssa\#STIR04893 & Lactose-binding lectin $\mathrm{I}-2$ precursor putative & 1.57 & 4.15 & 0.0419 \\
\hline Ssa\#S30264865 & Indoleamine-pyrrole 2,3-dioxygenase & 2.83 & 1.06 & 0.0444 \\
\hline Ssa\#S31981622 & Granzyme A & -5.31 & -4.33 & 0.0444 \\
\hline Ssa\#S35685718 & CD83 antigen precursor & 2.03 & 1.58 & 0.0462 \\
\hline Ssa\#KSS3969 & Leukocyte cell-derived chemotaxin 2 precursor (lect2) & 3.16 & 4.86 & 0.0486 \\
\hline \multicolumn{5}{|c|}{ Structural proteins (10\%) } \\
\hline Ssa\#STIR03004 & Troponin I, slow skeletal muscle & -202.28 & -135.03 & 0.0000 \\
\hline Ssa\#CK891024 & Vitelline envelope protein gamma & 1.07 & -15.43 & 0.0002 \\
\hline Ssa\#STIR02053 & Troponin I, slow skeletal muscle & -70.21 & -10.07 & 0.0005 \\
\hline Omy\#S34312003 & similar to Titin (Connectin) & -44.29 & -29.86 & 0.0013 \\
\hline Omy\#S15317515 & Type I keratin E7 & 5.08 & 2.43 & 0.0167 \\
\hline Ssa\#STIR05140 & Troponin I, slow skeletal muscle & -13.16 & -14.20 & 0.0124 \\
\hline Ssa\#STIR08802 & Myosin regulatory light chain 2, smooth muscle isoform & 1.55 & 1.34 & 0.0324 \\
\hline
\end{tabular}




\begin{tabular}{|c|c|c|c|c|}
\hline \multicolumn{5}{|c|}{ Miscellaneous/unknown function } \\
\hline Ssa\#S35519407 & Tetratricopeptide repeat protein 23 & 3.64 & 1.02 & 0.0012 \\
\hline Ssa\#S35584894 & NCK-associated protein 1-like & -1.82 & -1.70 & 0.0019 \\
\hline Ssa\#S35521859_S & FAM36A & 1.11 & 5.75 & 0.0047 \\
\hline Ssa\#STIR02307 & FAM36A & 1.16 & 3.31 & 0.0058 \\
\hline Ssa\#TC87798 & Envelope polyprotein & -1.83 & -1.03 & 0.0233 \\
\hline Ssa\#STIR20319 & TPA-induced transmembrane protein & -1.09 & 1.75 & 0.0233 \\
\hline Ssa\#TC110493_S & Beta-3-galactosyltransferase & 6.80 & 1.80 & 0.0233 \\
\hline Ssa\#S30270166 & Transmembrane protein 37 & 2.13 & 1.90 & 0.0254 \\
\hline Ssa\#STIR08658 & Tetraspanin-3 putative & -3.20 & -1.47 & 0.0312 \\
\hline Ssa\#S30293470 & Deoxyribonuclease gamma precursor & 2.19 & 2.85 & 0.0366 \\
\hline Ssa\#S35667723 & Dynein, cytoplasmic 1, light intermediate chain 2 & -1.90 & -1.48 & 0.0366 \\
\hline Ssa\#CX354464 & Retinol dehydrogenase 12 & 2.37 & 2.35 & 0.0373 \\
\hline Ssa\#S35582016 & Type I iodothyronine deiodinase & 1.51 & 1.74 & 0.0380 \\
\hline Ssa\#S35515630 & C7orf57 & 1.32 & 1.13 & 0.0380 \\
\hline Ssa\#STIR15617 & FAM36A & 1.02 & 4.60 & 0.0403 \\
\hline Ssa\#STIR31448 & osteopontin-like & -4.84 & -1.58 & 0.0419 \\
\hline Ssa\#STIR26263 & Anterior gradient protein 2 homolog precursor & 3.72 & 1.68 & 0.0419 \\
\hline Ssa\#TC65497_S & Adipocyte plasma membrane-associated protein & 2.50 & 1.64 & 0.0419 \\
\hline Ssa\#TC93681 & Ring finger protein 44 & -1.23 & -1.17 & 0.0455 \\
\hline Ssa\#EG819142 & Glutaminyl-peptide cyclotransferase-like & -1.11 & -1.49 & 0.0455 \\
\hline
\end{tabular}

Results were obtained by two-way ANOVA analysis ( $p<0.05$; fold change cut-off of 1.2) with Benjamini-Hochberg multiple testing correction. Transcripts (109 probes; $18 \%$ unknowns) are arranged by categories of biological function and, within these, by $p$-value. Indicated are also the probe names and the expression ratio determined separately between families with high vs low total lipid level, for each $n-3$ LC-PUFA grouping. Percentages of distribution of genes within each category do not include non-annotated probes, those representing the same gene or with a miscellaneous function.

and that mRNA levels correlate with enzymatic activity of this pathway [13,14], and so this appeared a likely mechanism that required specific investigation. Flesh was the target tissue for analysis of the n-3 LC-PUFA retention trait because salmon accumulate lipid reserves in muscle and this is the main product for human consumption, and so its composition will affect the healthpromoting properties of salmon. However, hepatic tissue was analyzed for effects on gene expression since the production of both LC-PUFA and the lipoproteins that transport them to the tissues takes place mainly in the liver [15].

The transcriptomic analysis revealed few effects of the n-3 LC-PUFA factor on metabolism in general and, in particular, a lack of effect on lipid metabolism genes, when the statistical analysis employed multiple testing correction. However, this correction is typically not used when examining effects of diet and genetic background on metabolic genes, which tend to show subtle, but physiologically relevant, changes $[9,11,16]$. Without multiple testing correction we were able to identify pathways of lipid metabolism that might be altered in response to this factor, although a clear mechanism for the observed inter-family differences in n-3 LC-PUFA content was not identified. Potential effects on lipid transport and lipoprotein metabolism were indicated by the presence of two apolipoprotein A4 transcripts (apoa4a and $b$ ), a low density lipoprotein (LDL) receptor-related protein $(l r p 1)$ and a lipoprotein lipase $(l p l)$ transcript in the microarray analysis, albeit these were not validated by RT-qPCR. In contrast, the RT-qPCR results clearly confirmed that the flesh n-3 LC-PUFA phenotype cannot be explained by transcriptional modulation of genes of LCPUFA biosynthesis and so other mechanisms must be in operation. One hypothesis might be that phenotypic differences between families originates from the presence of different alleles of fatty acyl desaturases and/or elongases encoding proteins with altered biological activity or specificity, as described for the nematode Caenorhabditis elegans [17].

\section{Effects of $n-3$ LC-PUFA flesh contents on hepatic cholesterol biosynthesis}

Within the lipid metabolism genes that were differentially expressed in the liver between fish showing higher or lower n-3 LC-PUFA contents in flesh, the category of 
Table 4 Lipid metabolism genes differentially expressed in the liver of fish having high or low flesh n-3 LC-PUFA contents

\begin{tabular}{|c|c|c|c|c|}
\hline \multirow[t]{2}{*}{ Probe name } & \multirow[t]{2}{*}{ Gene } & \multicolumn{2}{|c|}{ High/Low LC-PUFA } & \multirow[t]{2}{*}{ p-value } \\
\hline & & L Lipid (LH/LL) & H Lipid (HH/HL) & \\
\hline \multicolumn{5}{|c|}{ Cholesterol biosynthesis, metabolism and regulation } \\
\hline Omy\#S15288895 & Sterol-C4-methyl oxidase-like & 1.84 & 1.32 & 0.0033 \\
\hline Ssa\#STIR00031_3 & 7-dehydrocholesterol reductase (7dchr) & -1.55 & -1.48 & 0.0036 \\
\hline Ssa\#S30286041 & 7-dehydrocholesterol reductase (7dchr) & -1.48 & -1.42 & 0.0120 \\
\hline Omy\#CF752841 & $\begin{array}{l}\text { Sterol regulatory element-binding transcription } \\
\text { factor } 2 \text { (srebp2) }\end{array}$ & -1.71 & -1.31 & 0.0170 \\
\hline Ssa\#TC102141 & $\begin{array}{l}\text { Cytochrome P450, family 27, subfamily A, } \\
\text { polypeptide } 1\end{array}$ & -3.97 & -1.04 & 0.0192 \\
\hline Ssa\#STIR16974 & 7-dehydrocholesterol reductase ( $7 d c h r)$ & -1.46 & -1.31 & 0.0195 \\
\hline Ssa\#AM402497 & Hydroxymethylglutaryl-CoA synthase 1 & -2.39 & 1.21 & 0.0199 \\
\hline Ssa\#STIR00031_4 & 7-dehydrocholesterol reductase (7dchr) & -1.41 & -1.46 & 0.0212 \\
\hline Ssa\#STIR00098_4 & Isopentenyl-diphosphate delta isomerise (ipi) & -2.12 & -1.15 & 0.0228 \\
\hline Ssa\#S18867829 & 7-dehydrocholesterol reductase (7dchr) & -1.33 & -1.25 & 0.0228 \\
\hline Ssa\#DY741343 & Lanosterol 14-alpha demethylase & -1.42 & -1.28 & 0.0346 \\
\hline Omy\#S22913656 & Acetoacetyl-CoA synthetase & -1.77 & -2.52 & 0.0358 \\
\hline Ssa\#STIR00033_3 & Mevalonate kinase (mev) & -1.54 & -1.08 & 0.0393 \\
\hline Ssa\#CA064135 & Vigilin & 2.80 & -1.22 & 0.0423 \\
\hline Ssa\#DW582478 & Cytochrome P450, family 8, subfamily B, polypeptide 1 & -1.26 & -1.50 & 0.0449 \\
\hline Ssa\#STIR00102_3 & Squalene epoxidase & -2.11 & -1.36 & 0.0468 \\
\hline \multicolumn{5}{|c|}{ Glycerophospholipid synthesis } \\
\hline Ssa\#STIR39152_S & Lipid phosphate phosphohydrolase 2 (Ipp2) & 1.20 & 1.19 & 0.0177 \\
\hline Ssa\#KSS4003 & Chka protein & 1.18 & 1.53 & 0.0264 \\
\hline Ssa\#S35538062_S & Monoacylglycerol O-acyltransferase 1 (mgat) & 1.20 & 1.51 & 0.0303 \\
\hline Ssa\#S31963704 & Diacylglycerol O-acyltransferase homolog 2 & 1.41 & 1.02 & 0.0358 \\
\hline Ssa\#S48418830 & Phosphatidylglycerophosphate synthase 1 & -1.52 & -1.72 & 0.0369 \\
\hline \multicolumn{5}{|c|}{ Fatty acid synthesis } \\
\hline Ssa\#KSS4155 & Trans-2-enoyl-CoA reductase, mitochondrial precursor & -1.26 & -1.25 & 0.0440 \\
\hline \multicolumn{5}{|c|}{ Intracellular fatty acid transport } \\
\hline Ssa\#S35585414 & Acyl-CoA-binding protein & 10.47 & 1.65 & 0.0081 \\
\hline Ssa\#DY703528 & Fatty acid-binding protein, intestinal & -1.25 & -1.46 & 0.0331 \\
\hline Ssa\#CB509140 & Fatty acid-binding protein, intestinal & -1.20 & -1.35 & 0.0375 \\
\hline Ssa\#STIR04578 & Fatty acid-binding protein, heart & 1.22 & 1.47 & 0.0401 \\
\hline \multicolumn{5}{|l|}{ Lipid transport } \\
\hline Ssa\#CK898816 & Low density lipoprotein receptor-related protein 1 (Irp1) & -1.14 & -1.39 & 0.0341 \\
\hline Ssa\#S32008850 & Apolipoprotein A-IV precursor (apoa4a) & -1.43 & -1.01 & 0.0377 \\
\hline Omy\#BX318293 & Low density lipoprotein receptor-related protein 1 (Irp1) & -3.61 & -1.67 & 0.0404 \\
\hline Ssa\#S18866963 & Apolipoprotein A-IV precursor (apoa4b) & -1.39 & -1.43 & 0.0428 \\
\hline \multicolumn{5}{|l|}{ Lipid hydrolysis } \\
\hline Ssa\#S18887340 & Acyl-coenzyme A thioesterase 5 & 1.64 & 1.18 & 0.0007 \\
\hline Ssa\#STIR02708 & Isoamyl acetate-hydrolyzing esterase 1 homolog & 1.54 & 1.12 & 0.0013 \\
\hline Ssa\#DW007099 & $\begin{array}{l}\text { Similar to patatin-like phospholipase domain } \\
\text { containing } 7\end{array}$ & -3.75 & -2.37 & 0.0070 \\
\hline Ssa\#S31963297 & Acyl-CoA thioesterase 11 & 1.35 & 1.38 & 0.0097 \\
\hline Ssa\#STIR07750 & $\mathrm{N}$-acylsphingosine amidohydrolase 1 & -1.14 & -1.34 & 0.0122 \\
\hline
\end{tabular}




\begin{tabular}{|c|c|c|c|c|}
\hline Ssa\#STIR05034 & Isoamyl acetate-hydrolyzing esterase 1 homolog & 1.74 & 1.01 & 0.0208 \\
\hline Omy\#CA366823 & Acyl-coenzyme A thioesterase 3 & 1.33 & 2.17 & 0.0248 \\
\hline Ssa\#STIR22551 & Lipoprotein lipase (IpI) & 1.31 & 1.31 & 0.0346 \\
\hline \multicolumn{5}{|c|}{ Eicosanoid synthesis and metabolism } \\
\hline Ssa\#TC68569 & Thromboxane-A synthase (thas) & -1.33 & -1.67 & 0.0269 \\
\hline Ssa\#TC110080 & Phospholipase A2 (pla2g4) & 1.26 & 1.08 & 0.0296 \\
\hline Omy\#TC147730 & Prostaglandin 12 (prostacyclin) synthase (ptgis) & -2.53 & -4.25 & 0.0349 \\
\hline Ssa\#S35581706 & 15-hydroxyprostaglandin dehydrogenase & -1.51 & -1.09 & 0.0400 \\
\hline Ssa\#EG930234 & Arachidonate 5-lipoxygenase (alox5) & 1.91 & 1.55 & 0.0449 \\
\hline \multicolumn{5}{|c|}{ Regulation of lipid metabolism } \\
\hline Ssa\#TC112002 & Retinoid $X$ receptor beta & 1.00 & -19.09 & 0.0000 \\
\hline Ssa\#KSS2129 & Adiponectin receptor protein 1 & -1.30 & -1.31 & 0.0098 \\
\hline Ssa\#CA056493 & Angiopoietin-like 6 & -1.63 & -1.07 & 0.0290 \\
\hline Ssa\#S35490606 & Adiponectin, C1Q and collagen domain containing, like & 1.34 & 1.66 & 0.0441 \\
\hline Ssa\#S18888608 & Adiponectin receptor protein 1 & -1.38 & -1.40 & 0.0451 \\
\hline
\end{tabular}

Results were obtained by two-way ANOVA analysis ( $<<0.05$; fold change cut-off of 1.2) without multiple testing correction ( $n=1951$ total features). Transcripts are arranged by functional categories and, within these, by p-value. Indicated are also the probe names and the expression ratio between families determined separately for each total lipid level.

cholesterol biosynthesis and its regulation was the most apparent, based on the number of probes for interrelated genes present in this list, all with coordinated regulation indicating reduced cholesterol biosynthesis in salmon having higher flesh n-3 LC-PUFA. In addition, and inferred by the magnitude of change (i.e., fold-changes), effects were more pronounced in fish containing lower flesh lipid levels. These results were confirmed by quantifying the expression of three enzymes catalyzing steps in cholesterol biosynthesis (mev, ipi and 7dchr) as well as $\operatorname{srebp} 2$, a transcription factor that regulates cholesterol synthesis [18]. Furthermore, the RT-qPCR analysis indicated that this regulation was only associated with lower flesh lipid levels given that in the high lipid group only $7 d c h r$ was down-regulated. Therefore, this experiment confirmed previous studies suggesting an association between flesh adiposity and n-3 LC-PUFA in the regulation of cholesterol biosynthesis in Atlantic salmon families, with lean fish showing a higher responsiveness to n-3 LC-PUFA [8]. However, an important novel outcome of the present study was the demonstration that the previous results were not solely a consequence of a higher dietary intake of cholesterol supplied by a FO diet in contrast to a VO diet [11] but also resulted from higher incorporation and increased tissue levels of $n-3$ LC-PUFA. The likely explanation for these results is the role of $n-3$ LC-PUFA as regulators of gene transcription, including some implicated in cholesterol biosynthesis, mediated by srebp 2 [18-20]. Nonetheless, the mechanism for why this response was only observed when associated with low flesh lipid levels requires clarification. Recent studies showed that lean humans are also more responsive, in terms of plasma lipid and lipoprotein composition, to cholesterolreducing diets containing lower levels of saturated fatty acids and cholesterol than obese individuals, and several mechanisms have been proposed to explain this [21]. In the present case, the absolute, rather than the relative, level of n-3 LC-PUFA may be the determinant factor affecting gene transcription and, in the high lipid group, absolute levels of these fatty acids might have been sufficiently high to repress cholesterol biosynthesis genes, even at lower relative n-3 LC-PUFA contents (i.e., group HL). This hypothesis is supported by the RT-qPCR analysis comparing the families with regards to lipid level, HL/LL and HH/LH. In the HL/LL comparison, contrasting absolute n-3 LC-PUFA levels of 427 versus $363 \mathrm{mg} / 100 \mathrm{~g}$ flesh, there was downregulation of both ipi and srebp2 (-3.95 and -2.09 , respectively), whereas comparison of the families $\mathrm{HH} /$ $\mathrm{LH}$, containing 554 versus $468 \mathrm{mg} / 100 \mathrm{~g}$ flesh, showed no difference in the expression of the genes. Similarly, genes involved in lipoprotein metabolism, which are also regulated by LC-PUFA through different mechanisms [20], also showed more significant changes when comparing fatter and leaner salmon with lower LCPUFA levels, indicating that a similar regulatory mechanism might occur. Therefore, the present study is consistent with previous work identifying cholesterol and lipoprotein metabolism as pathways significantly and differentially affected by n-3 LC-PUFA depending on flesh adiposity [8]. 
Table 5 Validation of microarray results and expression of genes of interest in relation to the factor ' $n-3$ LC-PUFA level'

\begin{tabular}{|c|c|c|c|c|}
\hline \multirow[t]{2}{*}{ Gene } & \multicolumn{2}{|c|}{ Low Lipid LH/LL } & \multicolumn{2}{|c|}{ High Lipid HH/HL } \\
\hline & Microarray & RT-qPCR & Microarray & RT-qPCR \\
\hline
\end{tabular}

LC-PUFA biosynthesis

$\begin{array}{lrr}\Delta 5 \text { fad } & -1.19 & 1.17 \\ \Delta 6 \text { fad } & 1.13 & -1.21 \\ \text { elovl2 } & 1.14 & 1.06\end{array}$

Cholesterol biosynthesis

$\begin{array}{llrlr}\text { ipi } & -2.13 & \mathbf{- 3 . 9 2} & -1.15 & 1.32 \\ \text { mev } & -1.54 & -1.51 & -1.08 & 1.06 \\ \text { 7dchr } & -1.33 \text { to }-1.54 & \mathbf{- 1 . 4 7} & -1.25 \text { to }-1.47 & \mathbf{- 1 . 3 4} \\ \text { srebp2 } & -1.72 & \mathbf{- 1 . 6 8} & -1.30 & 1.60\end{array}$

Lipid transport and lipoprotein metabolism

\begin{tabular}{llrlr} 
Irpl & -1.14 to -3.57 & -1.36 & -1.39 to -1.67 & 1.24 \\
apoA4a & -1.43 & -1.09 & 1.00 & 1.32 \\
apoA4b & -1.39 & -1.48 & -1.43 & -1.10 \\
Ipl & 1.31 & 1.23 & 1.31 & 1.38 \\
Glycerophospholipid synthesis & & & \\
Ipp2 & 1.20 & -1.19 & 1.19 & 1.30 \\
mgat & 1.20 & 1.04 & 1.51 & 1.78 \\
Eicosanoid biosynthesis & & & \\
alox5 & 1.91 & $\mathbf{1 . 4 8}$ & 1.55 & $\mathbf{1 . 6 2}$ \\
pla2g4 & 1.26 & -1.08 & 1.08 & 1.06 \\
thas & -1.33 & -1.08 & -1.67 & $\mathbf{1 . 3 4}$ \\
ptgis & -2.53 & -1.25 & -4.25 & 1.27 \\
Immune response & & & \\
mal & -22.20 & $\mathbf{3 . 7 0}$ & -25.00 & $\mathbf{- 5 . 0 0}$ \\
cd13 & 4.40 & $\mathbf{5 . 9 8}$ & 3.00 & $\mathbf{2 . 5 1}$ \\
trim25 & 5.20 & $\mathbf{2 . 8 0}$ & 6.70 & $\mathbf{7 . 5 7}$ \\
lect2 & 4.60 & 1.92 & 7.10 & \\
\hline Valus reprent & & &
\end{tabular}

Values represent the expression ratios between high PUFA / low PUFA, for fish containing either low or high total lipid levels in their flesh, obtained by microarray analysis or RT-qPCR. Expression ratios in bold were significant by REST2008 analysis of RT-qPCR results.

Delta5 and 6 fatty acyl desaturases ( $\Delta 5$ fad and $\Delta 6$ fad); fatty acyl elongase (elov/2); isopentenyl-diphosphate isomerase (ipi); mevalonate kinase (mev); 7-dehydrocholesterol reductase ( $7 d c h r)$; sterol regulatory element-binding protein 2 (srebp2); low density lipoprotein receptor-related protein 1 (Irp1); apolipoprotein A-IV (apoa4a and apoa4b); lipoprotein lipase (Ip); lipid phosphate phosphohydrolase 2 (Ipp2); monoacylglycerol O-acyltransferase 1 (mgat); arachidonate 5-lipoxygenase (alox5); phospholipase A2 (pla2g4); thromboxane-A synthase (thas); prostaglandin 12 (prostacyclin) synthase (ptgis); myelin and lymphocyte protein (mal); c-c motif chemokine 13 precursor (ccl13); tripartite motif-containing protein 25 (trim25); leukocyte cell-derived chemotaxin 2 precursor (lect2).

Effects of total lipid level on lipid metabolism

Lipid level significantly affected expression of lipid metabolism genes, although effects were still relatively small ( $8 \%$ of all genes assigned to a biological function category). A noteworthy result was the down-regulation of elovl2 (confirmed by RT-qPCR) in salmon presenting higher flesh lipid, independent of LC-PUFA content. Elovl2 has substrate specificity towards LC-PUFA and is highly responsive to dietary $\mathrm{n}-3$ LC-PUFA levels in salmon [22]. However, the expression of this gene is often co-ordinately regulated with other genes of LC-PUFA biosynthesis, such as $\Delta 5 \mathrm{fad}$ and $\Delta 6 \mathrm{fad}$ [9], which was not the case here. Hence, the biological significance of this result is not clear and may indicate other roles of elovl2 in lipid metabolism. For instance, an association between overexpression of elovl2 and enhanced triacylglycerol synthesis and lipid droplet accumulation, as well as induction of PPAR $\gamma$ target genes, was shown in mouse preadipocyte cell lines [23]. In addition, elovl2 was up-regulated in the liver transcriptome of rats with nephrotic syndrome, a condition characterized by hyperlipidemia [24]. Elovl2 was only recently characterized in salmon [22], and this is the first indication of an association between its expression and lipid accumulation in a non-mammalian vertebrate, with results suggesting that

Table 6 Validation of microarray results and expression of genes of interest in relation to the factor 'Lipid level'

\begin{tabular}{|c|c|c|c|c|}
\hline \multirow[t]{2}{*}{ Gene } & \multicolumn{2}{|c|}{ Low n-3 LC-PUFA HL/LL } & \multicolumn{2}{|c|}{ High n-3 LC-PUFA HH/LH } \\
\hline & Microarray & RT-qPCR & Microarray & RT-qPCR \\
\hline \multicolumn{5}{|c|}{ LC-PUFA biosynthesis } \\
\hline$\Delta 5$ fad & & -1.03 & & 1.35 \\
\hline$\Delta 6 f a d$ & & 1.04 & & -1.32 \\
\hline elov/2 & -1.28 to -1.41 & -1.51 & -1.82 to -1.97 & -1.62 \\
\hline
\end{tabular}

Cholesterol biosynthesis

$\begin{array}{lrl}\text { ipi } & \mathbf{- 3 . 9 5} & 1.31 \\ \text { mev } & -1.40 & 1.14 \\ \text { 7dchr } & -1.01 & 1.09 \\ \text { srebp2 } & \mathbf{- 2 . 0 9} & 1.29\end{array}$

Lipid transport and lipoprotein metabolism

\begin{tabular}{|c|c|c|c|c|}
\hline \multicolumn{2}{|l|}{ |rp1 } & \multicolumn{2}{|l|}{-1.82} & -1.08 \\
\hline \multicolumn{2}{|c|}{$a p o A 4 a$} & \multicolumn{2}{|l|}{-1.37} & -0.95 \\
\hline \multicolumn{2}{|c|}{$a p o A 4 b$} & \multicolumn{2}{|l|}{-2.43} & -1.80 \\
\hline \multicolumn{2}{|l|}{$|p|$} & \multicolumn{2}{|l|}{-1.18} & -1.05 \\
\hline \multicolumn{5}{|c|}{ Immune response } \\
\hline$c f h$ & -1.33 to -1.56 & -1.24 & -1.56 to -1.69 & -1.31 \\
\hline $\operatorname{trim} 25$ & -5.64 & -2.09 & -4.34 & -2.33 \\
\hline lect2 & 3.16 & 1.23 & 4.86 & 4.84 \\
\hline
\end{tabular}

Values represent the expression ratios between high lipid / low lipid, for fish containing either low or high n-3 LC-PUFA levels in their flesh, obtained by microarray analysis or RT-qPCR. Expression ratios in bold were significant by REST2008 analysis of RT-qPCR results.

Delta5 and 6 fatty acyl desaturases ( $\Delta 5$ fad and $\Delta 6$ fad); fatty acyl elongase (elov/2); isopentenyl-diphosphate isomerase (ipi); mevalonate kinase (mev); 7-dehydrocholesterol reductase (7dchr); sterol regulatory element-binding protein 2 (srebp2); low density lipoprotein receptor-related protein 1 (Irp1); apolipoprotein A-IV (apoa4a and apoa4b); lipoprotein lipase (Ipl); complement factor $\mathrm{H}$ precursor (cfh); tripartite motif-containing protein 25 (trim25); leukocyte cell-derived chemotaxin 2 precursor (lect2). 
increased lipid level in salmon flesh repressed elovl2 expression independent of n-3 LC-PUFA level although this requires further investigation. Another gene downregulated at higher lipid levels was a mitochondrial acyl carrier protein, involved in acyl transfer steps, including roles in fatty acid synthesis and functioning of the electron transport chain [25], which could conceptually be responding to similar regulatory mechanisms affecting elovl2. In contrast, stearoyl-CoA desaturase, responsible for the synthesis of monounsaturated fatty acids from saturated precursors, was up-regulated in salmon with higher flesh lipid levels. This gene was positively correlated with fat accumulation in bovine skeletal muscle [26], consistent with up-regulation in salmon families with increased fat stores.

\section{Possible association between flesh n-3 LC-PUFA contents and immune response}

The predominance of immune response genes responding to total lipid level and, particularly, n-3 LC-PUFA contents in salmon flesh was unexpected. This was a true over-representation as GO enrichment analysis enabled identification of several GO terms related to regulation of immune and inflammatory responses in relation to the total lipid factor. However, as mentioned above, the transcriptomic comparison, although balanced for total lipid, was not balanced for viral disease resistance (specifically IPN in this case) and, as a consequence, higher contrast between families was imposed on the high lipid group (families HL and $\mathrm{HH}$ ) due to the fortuitous selection of family $\mathrm{HH}$ presenting a much higher viral resistance EBV. Nonetheless, if family $\mathrm{HH}$ biased the results of the twoway ANOVA we would expect a preponderance of immune-related genes to occur only when comparing these two families, presenting higher and lower flesh n-3 LC-PUFA contents at the higher lipid level. In order to assess this, t-tests were performed comparing separately the higher versus lower n-3 LC-PUFA families at each total lipid level, i.e., LH/LL and HH/HL. A Venn diagram contrasting the two t-test significant lists was then performed and when analyzing the genes that were similarly affected by n-3 LC-PUFA contents at both higher and lower total lipid level, a similar preponderance (33\%) of immune response genes was observed (Additional file 4). Finally, examination of the fold changes of immunerelated genes, indicating magnitude of effects, between families with higher and lower contents of n-3 LC-PUFA at either higher or lower total lipid levels (Tables 2 and 5), showed no clear evidence of the effect being more marked for the high lipid comparison, which is what would be expected if results were caused simply by inclusion of family $\mathrm{HH}$ in the transcriptomic analysis.

Hence, there is evidence to suggest that there may be some correlation between flesh n-3 LC-PUFA contents and immune response in the families analysed. An antiinflammatory role of n-3 LC-PUFA is well established in mammals and fish [27-29]. Immune cells are typically rich in arachidonic acid (ARA), the precursor for eicosanoids with a pro-inflammatory action, whereas EPA and DHA give rise to eicosanoids that are less biologically active, as well as to resolvins and protectins presenting anti-inflammatory properties [30]. Higher incorporation of n-3 LC-PUFA in biological membranes of immune cells can modulate immune responses in several ways [reviewed in 15,30-33]. They alter the production of inflammatory eicosanoid mediators of which they are precursors, directly affect the organization and properties of the immune cell membranes with effects on signalling pathways, phagocytic capacity and antigen presenting capability, and activate transcription of various genes involved in inflammatory responses. Therefore, families with higher tissue levels of n-3 LC-PUFA may show differential expression of immune response and inflammationrelated genes, as well as of genes involved in signalling and regulation of transcription (as observed in the present study). Furthermore, although liver is chiefly a metabolic organ, it has other physiological functions including removal of pathogens and antigens from the blood and modulation of immune responses, as well as the production of inflammatory mediators [34,35].

Related to the above, microarray analysis revealed the presence of several genes that intervene in eicosanoid synthesis and metabolism including phospholipase $\mathrm{A}_{2}$ (pla2), arachidonate 5-lipoxygenase (alox5), thromboxane-A synthase (thas), prostaglandin $\mathrm{I}_{2}$ synthase (ptgis) and 15hydroxyprostaglandin dehydrogenase [36]. However, RTqPCR only confirmed up-regulation of hepatic alox5 in families presenting higher flesh n-3 LC-PUFA and, given that alox 5 acts on LC-PUFA of both $n-3$ and $n-6$ series and that ARA levels generally accompanied the n-3 LCPUFA phenotype (Table 1), it cannot be ascertained whether this transcript was responding to higher levels of membrane ARA or EPA and hence if it would result in increased pro-inflammatory 4-series, or less potent 5series, leukotrienes [37].

The immune response genes whose expression was correlated with 'n-3 LC-PUFA' are mainly involved in the modulation of inflammatory processes and innate immune response to pathogens, which are particularly important in fish species and that can be easily compromised in aquaculture conditions [38]. We could speculate that the changes in expression may give enhanced protection from inflammation or pathological conditions in fish with higher n-3 LC-PUFA in their tissues. Up-regulation associated with high flesh n-3 LCPUFA was noted in expression of NACHT domain containing protein, tripartite motif-containing protein 25 (trim25), c-c motif chemokine 13 precursor (ccl13), 
leukocyte cell-derived chemotaxin 2 precursor (lect2), tissue factor pathway inhibitor a, pentraxin and cathepsin K. In contrast, down-regulation in the high n-3 LCPUFA families was observed for MHC class I (mostly in the high total lipid group), and for myelin and lymphocyte protein $(\mathrm{mal})$. NACHT domain containing proteins are pathogen-sensing molecules (recognizing intracellular pathogen-associated molecular patterns - PAMPs) implicated in early host defence, inflammation and innate immune signalling pathways in mammals [39], by activating transcription of MHC class II and the apoptotic pathway. The trim 25 protein is involved in antiviral innate immune responses through activation of signalling pathways leading to production of interferons and in teleost cells TRIM genes are induced in response to viral infections [40,41]. The ccl13 (also known as monocyte chemotactic protein 4) and lect2 proteins are both involved in inflammation, having roles in attracting monocytes and $\mathrm{T}$ lymphocytes in tissues exposed to exogenous pathogens, and have neutrophil chemotactic function [42,43]. Expression of lect 2 was increased in fish liver and spleen after bacterial infections [43]. Tissue factor pathway inhibitor inhibits the initial reactions of the blood coagulation cascade and modulates cell proliferation, and may protect vascular tissue in inflammatory conditions in mammals [44]. Cathepsin $\mathrm{K}$ mediates immune responses in cells, having a critical role in signalling events proximal to the Toll-like receptor 9 (TLR9) that has a fundamental role in pathogen recognition (recognizing PAMPs) and activation of mammalian innate immunity [45]. Finally, pentraxins are pattern recognition proteins of the innate immune system that play a role in the acute phase response, activating complement pathways to clear pathogens in both mammals and fish $[46,47]$. In this case, up-regulation of pentraxin in salmon with higher n-3 LC-PUFA in their flesh was only observed with high lipid levels. Similarly, down-regulation of the MHC class I transcript was observed only in the high lipid group. In mammalian studies, high LC-PUFA contents (EPA, DHA and ARA) reduced cell surface expression of MHC I, decreasing antigen presentation and altering Tcell signalling $[34,35]$. Therefore, the high IPN resistance genotype observed in family $\mathrm{HH}$ in later genetic evaluations of the families could potentially involve effects on both the complement pathway and T-cell mediated immunity, and involve co- or post-translational modification of proteins by N-linked glycosylation through upregulation of dolichyl-diphosphooligosaccharide-protein glycosyltransferase subunit 4 (Table 2; [48]). Given the high economic impact of IPN in salmonid culture, identification of genes potentially involved in the progression of the disease using transcriptomic approaches is already in progress [49]. Finally, down-regulation of mal, associated with T-cell differentiation and signal transduction [50], was observed at higher n-3 LC-PUFA levels.

As mentioned above, several immune response-related genes were also affected by the total lipid factor with results validated by RT-qPCR. However, we cannot exclude the possibility that this results from the strong correlation between total lipid levels and absolute LCPUFA contents, which makes it difficult to dissociate both factors.

\section{Conclusions}

It has been demonstrated earlier that LC-PUFA flesh content is a highly heritable trait [7], but the present study has shown that the underlying mechanisms do not appear to involve changes in the expression of lipid metabolism genes, including the LC-PUFA biosynthesis pathway. Other possible mechanisms, such as alleles with different biological activity, require investigation. The present study revealed an association between flesh adiposity and n-3 LC-PUFA in the regulation of cholesterol biosynthesis, which was down-regulated by higher $n-3$ LC-PUFA levels but only in the lean families. This response was not caused by dietary factors, given that the fish were all fed the same VO-based diet, and is most likely explained by variation in tissue n-3 LC-PUFA levels, regulating transcription of cholesterol metabolism genes through srebp2. Furthermore, the transcriptional repression of these genes may be sensitive to the absolute levels of these fatty acids in the tissues, which could explain the lack of regulation when comparing the families containing higher flesh lipid levels. It is likely that n-3 LC-PUFA exert similar roles in regulation of gene expression in fish as in mammals and, furthermore, fish might be a useful model to study important relationships between genetics, diet, adiposity/obesity and lipoprotein/cholesterol metabolism. However, unexpected differences were found in the expression of genes implicated in the modulation of inflammatory processes and innate immune response between families differing in lipid composition, both in terms of total lipid level and, particularly, n-3 LC-PUFA contents. Although the evidence is generally circumstantial it is important to clarify this association if flesh n-3 LC-PUFA level is included as a trait for genetic selection in Atlantic salmon breeding programmes. If such a relationship is confirmed, the data suggest that the underlying mechanism might involve anti-inflammatory actions of tissue n-3 LC-PUFA on the eicosanoid biosynthesis pathway (particularly affecting the lipoxygenase pathway), although direct effects through regulation of transcription of immune genes or more indirectly through changes in architecture and properties of immune cell membranes are also possible. 


\section{Methods}

\section{Feeding trial and sampling}

Fifty full-sib families selected from the 200 broodstock families of the Landcatch Natural Selection (LNS) Atlantic salmon breeding program (2005-strip yeargroup) were specifically selected for the feeding trial. On the basis of parental genetic evaluations, 25 high flesh lipid contrasting with 25 low flesh lipid families were identified, and 35 fish (initial weight, $100 \mathrm{~g}$ ) from each family were transferred and grown in communal seawater pens (Marine Harvest, Ardnish, Scotland). All fish were tagged with electronic transponders (PIT tags) to allow family identification while rearing in a common environment. After acclimation, the fish were grown for 12 weeks on the same low FM/high VO diet (Nutreco ARC, Stavanger, Norway) containing 25\% FM and 44\% plant meals and a VO blend including rapeseed oil/palm oil/camelina oil (2.5:1.5:1). At the end of the trial (378 g average weight), flesh samples (Norwegian Quality Cut) were collected, frozen on dry ice and stored at $-20^{\circ} \mathrm{C}$ until lipid analysis. Liver samples were also taken and stored at $-70^{\circ} \mathrm{C}$ for subsequent molecular analyses.

\section{Lipid analysis and choice of families for transcriptomic comparisons}

The 50 selected families were screened for their ability to retain and/or synthesize n-3 LC-PUFA when fed a low FM/high VO diet. De-boned and skinned flesh samples were combined into 3 pools per family for lipid analysis. Total lipids were extracted and determined gravimetrically from 1-2 g of pooled flesh [51]. Fatty acid methyl esters (FAME) were prepared by acidcatalyzed transesterification of total lipids [52]. Following purification, FAME were separated and quantified by gas-liquid chromatography as described in [9]. These data were used to select four families for transcriptomic analysis: two with equivalent high levels of lipid ' $H$ ', and two with equivalent low levels of lipid 'L'. Within each level of total lipid, two families with significantly contrasting ( $\mathrm{p}<0.05$ on Student's t-test; Graphpad Prism ${ }^{\mathrm{Tm}}$, version 4.0, Graphpad Software, San Diego, CA) relative n-3 LC-PUFA levels (similarly termed $\mathrm{H}$ and $\mathrm{L}$ ) were identified (Table 1).

\section{RNA extraction and purification}

Hepatic tissue $(200 \mathrm{mg}$ ) from ten individuals per family was rapidly homogenized in $2 \mathrm{ml}$ TRI Reagent (Ambion, Applied Biosystems, Warrington, U.K.). Total RNA was isolated, following manufacturer's instructions, and RNA quality and quantity was assessed by gel electrophoresis and spectrophotometry (NanoDrop ND-1000, Thermo Scientific, Wilmington, U.S.A.), respectively. Equal amounts $(50 \mu \mathrm{g})$ of total RNA were pooled from two individuals to produce five biological replicates per family, which were further purified by mini spin-column purification (RNeasy Mini Kit, Qiagen, Crawly, U.K.).

\section{Microarray hybridization and analysis}

A custom-made Atlantic salmon oligoarray with $44 \mathrm{~K}$ features per array on a four-array-per-slide format (Agilent Technologies, Cheshire, U.K.), with experimental features printed singly was used [described more fully in 53]. The probes were co-designed at the Institute of Aquaculture, University of Stirling, U.K. and Nofima, Norway, with array design available in the EBI ArrayExpress database (http://www.ebi.ac.uk/arrayexpress/ arrays/browse.html) under accession number A-MEXP2065. The features were mainly derived from a core set of Atlantic salmon Unigenes (NCBI) supplemented with other unique cDNAs derived from Genbank and the Atlantic Salmon Gene Index (http://compbio.dfci.harvard. edu/tgi/tgipage.html). Probe annotations were derived from Blastx comparisons across four protein databases, as detailed elsewhere [54]. The entire experiment comprised 20 hybridizations ( 5 slides): 4 groups (families) $\times 5$ biological replicates (pools of 2 individuals each).

Indirect labelling was employed in preparing the microarray targets, as described in detail previously [8]. Antisense amplified RNA (aRNA) was produced from $500 \mathrm{ng}$ of each total RNA purification reaction using the Amino Allyl MessageAmpTM II aRNA Amplification Kit (Ambion, Applied Biosystems), following the manufacturer's methodology followed by Cy3 or Cy5 fluor (PA23001 or PA25001, GE HealthCare) incorporation through a dye-coupling reaction.

The hybridizations were performed using SureHyb hybridisation chambers (Agilent) in a DNA Microarray Hybridisation Oven (Agilent). Sample order was semirandomized, with one replicate per experimental group being loaded into each slide. Each biological replicate pool was co-hybridized in a two-dye experiment with a single pooled reference sample. This pooled reference comprised equal quantitites of aRNA from all 20 biological replicate pools. Microarry manufacturer's instructions were followed. Briefly, for each hybridization, $825 \mathrm{ng}$ of Cy3-labelled experimental biological replicate and Cy5-labelled reference pool were combined. A fragmentation master mix containing $10 \times$ blocking agent (Agilent), 25x fragmentation buffer (Agilent) and nuclease-free water, was dispensed into the Cy-dyes mix. After incubating in the dark at $60^{\circ} \mathrm{C}$ for 30 mins, $2 \times$ GE Hybridization buffer (pre-heated to $37^{\circ} \mathrm{C}$; Agilent) was added, contents gently mixed, spun at $16 \mathrm{~K} g$ for 1 min and finally kept on ice until loaded onto the microarray slides. Hybridization was carried out in the oven rotator (Agilent) at $65^{\circ} \mathrm{C}$ and $10 \mathrm{rpm}$ for $17 \mathrm{~h}$. Post-hybridization washes were carried out in EasyDipTM Slide staining containers (Canemco Inc., Quebec, 
Table 7 Primers used for RT-qPCR analyses

\begin{tabular}{|c|c|c|c|c|c|c|}
\hline Transcript & Primer sequence $\left(5^{\prime}-3^{\prime}\right)$ & Fragment & $\mathrm{Ta}$ & Efficiency & Accession No. & Source \\
\hline \multirow[t]{2}{*}{$\overline{\Delta 5 \mathrm{fad}}$} & GTGAATGGGGATCCATAGCA & $192 \mathrm{bp}$ & $56^{\circ} \mathrm{C}$ & 0,945 & AF478472 ${ }^{1}$ & {$[9]$} \\
\hline & AAACGAACGGACAACCAGA & & & & & \\
\hline \multirow[t]{2}{*}{$\Delta 6 f a d \_a$} & CCCCAGACGTTTGTGTCAG & $181 \mathrm{bp}$ & $56^{\circ} \mathrm{C}$ & 0,928 & AY458652 ${ }^{1}$ & {$[9]$} \\
\hline & CCTGGATTGTTGCTTTGGAT & & & & & \\
\hline \multirow[t]{2}{*}{ elovl2 } & CGGGTACAAAATGTGCTGGT & $145 \mathrm{bp}$ & $60^{\circ} \mathrm{C}$ & 0,926 & TC91192 $^{2}$ & [24] \\
\hline & TCTGTTTGCCGATAGCCATT & & & & & \\
\hline \multirow[t]{2}{*}{ ipi } & ACAGCCCTATGGTTATGTGTCATCTC & $230 \mathrm{bp}$ & $60^{\circ} \mathrm{C}$ & 0,985 & CK875291 ${ }^{1}$ & [11] \\
\hline & CAAGGTGAGGCGAATGTTTGAAC & & & & & \\
\hline \multirow[t]{2}{*}{ mev } & CCCTTAATCAGGGTCCCAAT & $247 \mathrm{bp}$ & $60^{\circ} \mathrm{C}$ & 0,910 & DW005667 ${ }^{1}$ & [11] \\
\hline & GGTGCTGGTTGATGTCAATG & & & & & \\
\hline \multirow[t]{2}{*}{$7 d c h r$} & CTTCTGGAATGAGGCATGGT & $230 \mathrm{bp}$ & $60^{\circ} \mathrm{C}$ & 0,977 & TC99602 $^{2}$ & [11] \\
\hline & ACAGGTCCTTCTGGTGGTTG & & & & & \\
\hline \multirow[t]{2}{*}{ srebp2 } & GACAGGCACAACACAAGGTG & $215 \mathrm{bp}$ & $60^{\circ} \mathrm{C}$ & 0,887 & DY733476 ${ }^{1}$ & [11] \\
\hline & CAGCAGGGGTAAGGGTAGGT & & & & & \\
\hline \multirow[t]{2}{*}{ Irp1 } & ACCAACCGCATCTACTGGAC & 204 bp & $60^{\circ} \mathrm{C}$ & 0,996 & CK898816 $^{1}$ & New design \\
\hline & CAGATTACCAGCCACCCAGT & & & & & \\
\hline \multirow[t]{2}{*}{$a p o A 4 a$} & CCCAAACCAACACCACTCCT & $150 \mathrm{bp}$ & $60^{\circ} \mathrm{C}$ & 0,997 & BT047465 ${ }^{1}$ & New design \\
\hline & GGTTTATATTTCTCACCCTGCAC & & & & & \\
\hline \multirow[t]{2}{*}{$a p \circ A 4 b$} & CTCTTGCCCTCTTGATGACTG & $154 \mathrm{bp}$ & $60^{\circ} \mathrm{C}$ & 0,918 & BT047267 $^{1}$ & New design \\
\hline & TGACTCATCAGAGCCAATTCA & & & & & \\
\hline \multirow[t]{2}{*}{$|p|$} & AGGGCGTTAATCCATGTCAG & $223 \mathrm{bp}$ & $60^{\circ} \mathrm{C}$ & 0,917 & TC84899 ${ }^{2}$ & [8] \\
\hline & GACCTTTCAAAAGGGCATGA & & & & & \\
\hline \multirow[t]{2}{*}{ lpp2 } & TCCGGAAGAACTCGCAATAC & $174 \mathrm{bp}$ & $60^{\circ} \mathrm{C}$ & 0,926 & NM_001140716 ${ }^{1}$ & [9] \\
\hline & ACATCACGTCCACCAAGACA & & & & & \\
\hline \multirow[t]{2}{*}{ mgat } & TTAACCCAAAGATGCTGCAA & $157 \mathrm{bp}$ & $60^{\circ} \mathrm{C}$ & 0,977 & EG824440 ${ }^{1}$ & New design \\
\hline & CACGCAGTTGTCAGTGGTTT & & & & & \\
\hline \multirow[t]{2}{*}{ alox5 } & TATCTCCСTCTCCCTCAGTCC & $155 \mathrm{bp}$ & $56^{\circ} \mathrm{C}$ & 0,987 & CX727592 ${ }^{1}$ & {$[57]$} \\
\hline & GGTCAGCAGTGCCATCA & & & & & \\
\hline \multirow[t]{2}{*}{ pla2g4 } & GTCGCTGGCTGGAGCTGTGG & $138 \mathrm{bp}$ & $60^{\circ} \mathrm{C}$ & 0,998 & NM_001141333 ${ }^{1}$ & New design \\
\hline & AGCCCTATGGGCCCTGGTCA & & & & & \\
\hline \multirow[t]{2}{*}{ thas } & TGTTCACACGGACCTGATTC & $150 \mathrm{bp}$ & $60^{\circ} \mathrm{C}$ & 0,986 & NM_001165312 ${ }^{1}$ & New design \\
\hline & GACCGGATCGTCATTCTGTT & & & & & \\
\hline \multirow[t]{2}{*}{ ptgis } & GCGTGTTGTGGTCATTACG & $247 \mathrm{bp}$ & $60^{\circ} \mathrm{C}$ & 0,836 & GE778709 ${ }^{1}$ & New design \\
\hline & TTCCCTTAGCAAGGTCTGGA & & & & & \\
\hline \multirow[t]{2}{*}{ mal } & GGCCTCAGTCAAAGAGGAGA & $156 \mathrm{bp}$ & $60^{\circ} \mathrm{C}$ & 0,946 & NM_001141320 ${ }^{1}$ & New design \\
\hline & GGGGAGTGCACACTITAGGA & & & & & \\
\hline \multirow[t]{2}{*}{ ccl13 } & CGAGGATCCCTCTTCAACAA & $178 \mathrm{bp}$ & $60^{\circ} \mathrm{C}$ & 0,996 & EG831431 ${ }^{1}$ & New design \\
\hline & ATCGTCGACTAGGCAGCAGT & & & & & \\
\hline \multirow[t]{2}{*}{$\operatorname{trim} 25$} & GCAGGGTCCTATCTCATCCA & $215 \mathrm{bp}$ & $60^{\circ} \mathrm{C}$ & 0,951 & BT048046 $^{1}$ & New design \\
\hline & GGACTGGACCTIITTATTCTCTCA & & & & & \\
\hline \multirow[t]{2}{*}{ lect2 } & CTGTGTTGTCAGAGTGCGAGATGGT & $150 \mathrm{bp}$ & $60^{\circ} \mathrm{C}$ & 0,996 & BT050009 ${ }^{1}$ & [58] \\
\hline & TACACACAATGTCCAGGCCCTGA & & & & & \\
\hline$c f h$ & TGTGATGATGGAGAGATGCAG & $193 \mathrm{bp}$ & $60^{\circ} \mathrm{C}$ & 0,966 & TC141997 ${ }^{2}$ & New design \\
\hline
\end{tabular}


Table 7 Primers used for RT-qPCR analyses (Continued)

\begin{tabular}{|c|c|c|c|c|c|c|}
\hline \multicolumn{7}{|c|}{ Reference genes* } \\
\hline \multirow[t]{2}{*}{ elf-1a } & CTGCCCCTCCAGGACGTTTACAA & $175 \mathrm{bp}$ & $60^{\circ} \mathrm{C}$ & 1.000 & AF321836 ${ }^{1}$ & [11] \\
\hline & CACCGGGCATAGCCGATTCC & & & & & \\
\hline \multirow[t]{2}{*}{$\beta$-actin } & ACATCAAGGAGAAGCTGTGC & $141 \mathrm{bp}$ & $56^{\circ} \mathrm{C}$ & 0.939 & AF012125 ${ }^{1}$ & [11] \\
\hline & GACAACGGAACCTCTCGTTA & & & & & \\
\hline
\end{tabular}

Canada). After disassembling the array-gasket sandwiches submersed in wash buffer 1 (Agilent) at room temperature, the microarray slides were incubated in wash buffer 1 for $1 \mathrm{~min}$ at $31^{\circ} \mathrm{C}$ in a Stuart Orbital Incubator S150 rotating at $150 \mathrm{rpm}$, and then a further $1 \mathrm{~min}$ at $31^{\circ} \mathrm{C}$ at $150 \mathrm{rpm}$ in wash buffer 2 (Agilent). A final dip in wash buffer 2 at room temperature was performed, after which the slides were dried by centrifugation $(500$ $\mathrm{xg}$ for 6 mins) and kept in a desiccator and in the dark until scanned, the same day.

Scanning was performed at $5 \mu \mathrm{m}$ resolution using an Axon GenePix 4200AL Scanner (MDS Analytical Technologies, Wokingham, Berkshire, U.K.). Laser power was kept constant (50\%) and the "auto PMT" function within the acquisition software (v.4) was enabled to adjust PMT for each channel such that less than $0.1 \%$ of features were saturated and that the mean intensity ratio of the Cy3 and Cy5 signals was close to one. Agilent Feature Extraction Software (v 9.5) was used to identify features and extract fluorescence intensity values from the resultant TIF images. Analysis of the intensity values was performed in the GeneSpring GX version 11 analysis platform (Agilent Technologies, Wokingham, Berkshire, U.K.). All intensity values $<0.1$ were set to equal 0.1 followed by a Lowess normalization. After removing control features, four quality filtering steps were carried out sequentially using a range of quality control metrics produced by the Agilent Feature Extraction software to remove features that were saturated, non uniform, population outliers and spots non-significantly different from background. This gave a final list of 32,566 probes that were eligible for statistical analysis. Experimental annotation complied fully with minimum information about a microarray experiment (MIAME) guidelines [55]. The experimental hybridizations and further methodological details are archived on the EBI ArrayExpress database under accession number E-TABM-1204.

Normalized and quality-filtered fluorescence intensity data was analysed in GeneSpring GX v11 by two-way ANOVA, which examined the explanatory power of the variables 'total lipid' and ' $\mathrm{n}-3$ LC-PUFA' and the interaction between the two, at a significance level of 0.05 and expression ratio (i.e., fold change) cut-off of 1.2.
Two sets of analysis were performed, with or without Benjamini-Hochberg multiple testing correction. In the set with multiple testing correction, GO enrichment analysis was performed at a significance level of 0.05 .

\section{RT-qPCR}

Expression of selected genes found by microarray analysis to be significantly affected by either 'total lipid' or 'n-3 LC-PUFA' content was quantified by RT-qPCR. In addition, the expression of two fatty acyl desaturases ( $\Delta 5 f a d$ and $\Delta 6 f a d)$ and one elongase (elovl2) that are typically responsive to dietary n-3 LC-PUFA was determined. Primers were designed using Primer3 software (http://biotools.umassmed.edu/bioapps/primer3_www.cgi) (Table 7). Two reference genes, elongation factor- $1 \alpha$ (elf$1 \alpha)$ and $\beta$-actin, were also quantified.

For RT-qPCR, $2 \mu \mathrm{g}$ of column-purified total RNA per sample was reverse transcribed into cDNA using the High-Capacity cDNA RT kit (Applied Biosystems, Paisley, U.K.), following manufacturer's instructions, but using a mixture of the random primers $(1.5 \mu \mathrm{l}$ as supplied) and anchored oligo-dT $(0.5 \mu \mathrm{l}$ at $400 \mathrm{ng} / \mu \mathrm{l}$, Eurofins MWG Operon, Ebersberg, Germany). Negative controls (containing no enzyme) were performed to check for genomic DNA contamination. A similar amount of cDNA was pooled from all samples and the remaining cDNA was then diluted 20 -fold with water. RT-qPCR analysis used relative quantification with the amplification efficiency of the primer pairs being assessed by serial dilutions of the cDNA pool. Amplifications were carried out in duplicate (Quantica, Techne, Cambridge, U.K.) in a final volume of $20 \mu$ l containing $5 \mu \mathrm{l}$ or $2 \mu \mathrm{l}$ (for more highly expressed genes) diluted (1/ 20) $\mathrm{cDNA}, 0.5 \mu \mathrm{M}$ of each primer $(0.4 \mu \mathrm{M}$ for lect 2$)$ and $10 \mu \mathrm{l}$ AbsoluteTM QPCR SYBR ${ }^{\circledR}$ Green mix (ABgene). Amplifications were carried out with a systematic negative control (NTC-non template control, containing no cDNA). The RT-qPCR profiles contained an initial activation step at $95^{\circ} \mathrm{C}$ for $15 \mathrm{~min}$, followed by 30 to 40 cycles: $15 \mathrm{~s}$ at $95^{\circ} \mathrm{C}, 15 \mathrm{~s}$ at the specific primer pair annealing temperature (Ta; Table 7) and $15 \mathrm{~s}$ at $72^{\circ} \mathrm{C}$. After the amplification phase, a melt curve of $0.5^{\circ} \mathrm{C}$ increments from $75^{\circ} \mathrm{C}$ to $90^{\circ} \mathrm{C}$ was performed, enabling 
confirmation of the amplification of a single product in each reaction. Non-occurrence of primer-dimer formation in the NTC was verified. RT-qPCR product sizes and presence of single bands were checked by agarose gel electrophoresis. Additionally, sequencing of amplicons corresponding to new primer designs enabled the confirmation of identities and presence of single sequences for all genes except for trim 25 , as the sequencing result was of insufficient quality to conclude on the presence of a single gene product, and $\operatorname{lrp} 1$, for which results were indicative of quantification of a highly similar, recently duplicated, gene.

Results were analyzed by the $\Delta \Delta \mathrm{Ct}$ method using the relative expression software tool (REST 2009, http:// www.gene-quantification.info/), which employs a pair wise fixed reallocation randomization test $(10,000$ randomizations) with efficiency correction [56], to determine the statistical significance of expression ratios (or gene expression fold-changes) between two treatments.

\section{Genetic evaluations of traits used in the salmon breeding program}

Parental evaluations were confirmed by subsequent analysis of family sibs (at harvest weight, some 1 year after the present study) for a range of traits upon which the breeding program families are under active selection including flesh lipid composition parameters (total lipid) as well as EBVs for weight at harvest, precocious maturation, flesh colour, sealice resistance and resistance to a viral infection (IPN).

\section{Additional files}

Additional file 1: Figure S1. Relationship between total lipid level and n-3 LC-PUFA content.

Additional file 2: Table S1. Gene Ontology terms showing significant enrichment in the list of features affected by the 'total lipid' factor.

Additional file 3: Figure S2. Distribution of IPN resistance scores in relation to flesh lipid phenotypes.

Additional file 4: Table S2. Genes similarly regulated in pair-wise comparisons of families containing $\mathrm{H}$ and L n-3 LC-PUFA flesh contents, at each one of the total lipid levels.

Competing interests

The authors declare that they have no competing interests.

\section{Author's contributions}

SM performed laboratory analyses and data analysis. DRG was responsible for family selection. JBT supported the microarray analysis. SM wrote the first draft of the manuscript, followed by contributions from remaining authors. SM, JBT and DRT planned and coordinated the research. DRG, JGB and DRT were project leaders. All authors read and approved the final manuscript.

\section{Acknowledgements}

This study was funded by the EU FP6 IP "AQUAMAX" (Sustainable Aquafeeds to Maximise the Health Benefits of Farmed Fish for Consumers; 016249-2). SM was supported by Fundação para a Ciência e a Tecnologia, Portugal (SFRH/BPD/64679/2009). Technical assistance from Jacquie Ireland in microarray hybridizations is deeply appreciated.

\section{Author details}

'Institute of Aquaculture, University of Stirling, Stirling, FK9 4LA Scotland, UK 'Landcatch Natural Selection Ltd, Cooperage Way, Alloa, FK10 3LP Scotland, UK.

Received: 21 November 2011 Accepted: 18 July 2012

Published: 20 August 2012

\section{References}

1. FAO: The State of World Fisheries and Aquaculture 2008. Rome: Food and Agriculture Organization of the United Nations; 2009.

2. Tacon AGJ, Metian M: Global overview on the use of fish meal and fish oil in industrially compounded aquafeeds: Trends and future prospects. Aquaculture 2008, 285:146-158.

3. Calder PC, Yaqoob P: Omega-3 polyunsaturated fatty acids and human health outcomes. Biofactors 2009, 3:5266-272.

4. Menoyo D, López-Bote CJ, Obach A, Bautista JM: Effect of dietary fish oil substitution with linseed oil on the performance, tissue fatty acid profile, metabolism, and oxidative stability of Atlantic salmon. J Anim Sci 2005, 83:2853-2862

5. Gjedrem T, Baranski M: Selective Breeding in Aquaculture: an Introduction [Series: Reviews: Methods and Technologies in Fish Biology and Fisheries, Vol. 10]. London: Springer Science + Business Media B.V; 2009.

6. Schlechtriem C, Bron JE, Tocher DR: Inter-individual variation in total lipid fatty acid compositions of flesh of Atlantic salmon smolts fed diets containing fish oil or vegetable oil. Aquaculture Res 2007, 38:1045-1055.

7. Leaver MJ, Taggart JB, Villeneuve L, Bron JE, Guy DR, Bishop SC, Houston RD, Matika O, Tocher DR: Heritability and mechanisms of n-3 long chain polyunsaturated fatty acid deposition in the flesh of Atlantic salmon. Comp Biochem Physiol Part D Genomics Proteomics 2011, 6:62-69.

8. Morais S, Pratoomyot J, Torstensen BE, Taggart JB, Guy DR, Bell JG, Tocher DR: Diet $\times$ genotype interactions in hepatic cholesterol and lipoprotein metabolism in Atlantic salmon (Salmo salar) in response to replacement of dietary fish oil with vegetable oil. Br J Nutr 2011 , 106:1457-1469.

9. Morais S, Pratoomyot J, Taggart JB, Bron JE, Guy DR, Bell JG, Tocher DR: Genotype-specific responses in Atlantic salmon (Salmo salar) subject to dietary fish oil replacement by vegetable oil: a liver transcriptomic analysis. BMC Genomics 2011, 12:255.

10. LeBlanc F, Laflamme M, Gagné N: Genetic markers of the immune response of Atlantic salmon (Salmo salar) to infectious salmon anemia virus (ISAV). Fish Shellfish Immunol 2010, 29:217-232.

11. Leaver MJ, Villeneuve LA, Obach A, Jensen L, Bron JE, Tocher DR, Taggart JB: Functional genomics reveals increases in cholesterol biosynthetic genes and highly unsaturated fatty acid biosynthesis after dietary substitution of fish oil with vegetable oils in Atlantic salmon (Salmo salar). BMC Genomics 2008, 9:299.

12. Houston RD, Haley CS, Hamilton A, Guy DR, Tinch AE, Taggart JB, McAndrew BJ, Bishop SC: Major quantitative trait loci affect resistance to infectious pancreatic necrosis in Atlantic salmon (Salmo salar). Genetics 2008, 178:1109-1115.

13. Zheng X, Tocher DR, Dickson CA, Bell JG, Teale AJ: Effects of diets containing vegetable oil on expression of genes involved in highly unsaturated fatty acid biosynthesis in liver of Atlantic salmon (Salmo salar). Aquaculture 2004, 236:467-483.

14. Zheng X, Torstensen BE, Tocher DR, Dick JR, Henderson RJ, Bell JG: Environmental and dietary influences on highly unsaturated fatty acid biosynthesis and expression of fatty acyl desaturase and elongase genes in liver of Atlantic salmon (Salmo salar). Biochim Biophys Acta 2005, 1734:13-24

15. Galli $C$, Calder PC: Effects of fat and fatty acid intake on inflammatory and immune responses: a critical review. Ann Nutr Metab 2009, 55:123-139.

16. Kolditz Cl, Paboeuf $G$, Borthaire $M$, Esquerré $D$, SanCristobal $M$, Lefèvre F, Médale F: Changes induced by dietary energy intake and divergent selection for muscle fat content in rainbow trout (Oncorhynchus mykiss), assessed by transcriptome and proteome analysis of the liver. BMC Genomics 2008, 9:506.

17. Watts JL, Browse J: Genetic dissection of polyunsaturated fatty acid synthesis in Caenorhabditis elegans. Proc Natl Acad Sci U S A 2002, 99:5854-5859 
18. Sakakura $Y$, Shimano H, Sone H, Takahashi A, Inoue $K$, Toyoshima H, Suzuki S, Yamada N: Sterol regulatory element-binding proteins induce an entire pathway of cholesterol synthesis. Biochem Biophys Res Commun 2001, 286:176-183.

19. Jump DB, Botolin D, Wang Y, Xu J, Christian B, Demeure O: Fatty acid regulation of hepatic gene transcription. J Nutr 2005, 135:2503-2506.

20. Sampath H, Ntambi JM: Polyunsaturated fatty acid regulation of genes of lipid metabolism. Annu Rev Nutr 2005, 25:317-340.

21. Flock MR, Green MH, Kris-Etherton PM: Effects of adiposity on plasma lipid response to reductions in dietary saturated fatty acids and cholesterol. Adv Nutr 2011, 2:261-274.

22. Morais S, Monroig O, Zheng X, Leaver MJ, Tocher DR: Highly unsaturated fatty acid synthesis in Atlantic salmon: characterization of ELOVL5- and ELOVL2-like elongases. Mar Biotechnol 2009, 1:627-639.

23. Kobayashi T, Zadravec D, Jacobsson A: ELOVL2 overexpression enhances triacylglycerol synthesis in 3 T3-L1 and F442A cells. FEBS Lett 2007, 581:3157-3163

24. Zhou $Y$, Zhang $X$, Chen L, Wu J, Dang H, Wei M, Fan $Y$, Zhang $Y$, Zhu Y, Wang N, Breyer MD, Guan Y: Expression profiling of hepatic genes associated with lipid metabolism in nephrotic rats. Am J Physiol Renal Physiol 2008, 295:F662-671

25. Feng D, Witkowski A, Smith S: Down-regulation of mitochondrial acyl carrier protein in mammalian cells compromises protein lipoylation and respiratory complex I and results in cell death. J Biol Chem 2009, 284:11436-11445.

26. Jiang Z, Michal JJ, Tobey DJ, Daniels TF, Rule DC, Macneil MD: Significant associations of stearoyl-CoA desaturase (SCD1) gene with fat deposition and composition in skeletal muscle. Int J Biol Sci 2008, 4:345-351.

27. Montero D, Grasso V, Izquierdo MS, Ganga R, Real F, Tort L, Caballero MJ, Acosta F: Total substitution of fish oil by vegetable oils in gilthead sea bream (Sparus aurata) diets: effects on hepatic Mx expression and some immune parameters. Fish Shellfish Immunol 2008, 24:147-155.

28. Oxley A, Jolly C, Eide T, Jordal AE, Svardal A, Olsen RE: The combined impact of plant-derived dietary ingredients and acute stress on the intestinal arachidonic acid cascade in Atlantic salmon (Salmo salar). $\mathrm{Br}$ Nutr 2010, 103:851-861.

29. Estensoro I, Benedito-Palos L, Palenzuela O, Kaushik S, Sitjà-Bobadilla A Pérez-Sánchez J: The nutritional background of the host alters the disease course in a fish-myxosporean system. Vet Parasitol 2011 175:141-150.

30. Yaqoob P: Mechanisms underlying the immunomodulatory effects of $n-3$ PUFA. Proc Nutr Soc 2010, 69:311-315

31. Calder PC: Dietary modification of inflammation with lipids. Proc Nutr SoC 2002, 61:345-358.

32. Shaikh SR, Edidin M: Polyunsaturated fatty acids, membrane organization, T cells, and antigen presentation. Am J Clin Nutr 2006, 84:1277-1289.

33. Yaqoob P, Calder PC: Fatty acids and immune function: new insights into mechanisms. Br J Nutr 2007, 98(Suppl 1):S41-45.

34. Kmieć Z: Cooperation of liver cells in health and disease. Adv Anat Embryol Cell Biol 2001, 161:II-XIII. 1-151.

35. Knolle PA, Gerken G: Local control of the immune response in the liver. Immunol Rev 2000, 174:21-34.

36. Smith WL: The eicosanoids and their biochemical mechanisms of action Biochem J 1989, 259:315-324

37. Lee TH, Mencia-Huerta JM, Shih C, Corey EJ, Lewis RA, Austen KF: Effects of exogenous arachidonic, eicosapentaenoic, and docosahexaenoic acids on the generation of 5-lipoxygenase pathway products by ionophoreactivated human neutrophils. J Clin Invest 1984, 74:1922-1933.

38. Ellis AE: Innate host defense mechanisms of fish against viruses and bacteria. Dev Comp Immunol 2001, 25:827-839.

39. Stein C, Caccamo M, Laird G, Leptin M: Conservation and divergence of gene families encoding components of innate immune response systems in zebrafish. Genome Biol 2007, 8:R251.

40. Munir M: TRIM proteins: another class of viral victims. Sci Signal 2010, 3:jc2.

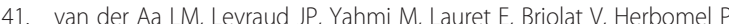
Benmansour A, Boudinot P: A large new subset of TRIM genes highly diversified by duplication and positive selection in teleost fish. BMC Biol 2009, 7:7.

42. Uguccioni M, Loetscher P, Forssmann U, Dewald B, Li H, Lima SH, Li Y, Kreider B, Garotta G, Thelen M, Baggiolini M: Monocyte chemotactic protein 4 (MCP-4), a novel structural and functional analogue of MCP-3 and eotaxin. J Exp Med 1996, 183:2379-2384.

43. Li MY, Chen J, Shi YH: Molecular cloning of leucocyte cell-derived chemotaxin-2 gene in croceine croaker (Pseudosciaena crocea). Fish Shellfish Immunol 2008, 24:252-256.

44. Kato H: Regulation of functions of vascular wall cells by tissue facto pathway inhibitor: basic and clinical aspects. Arterioscler Thromb Vasc Biol 2002, 22:539-548.

45. Asagiri M, Hirai T, Kunigami T, Kamano S, Gober HJ, Okamoto K, Nishikawa K, Latz E, Golenbock DT, Aoki K, Ohya K, Imai Y, Morishita Y, Miyazono K, Kato S, Saftig P, Takayanagi H: Cathepsin K-dependent toll-like receptor 9 signaling revealed in experimental arthritis. Science 2008, 319:624-627.

46. Agrawal A, Singh PP, Bottazzi B, Garlanda C, Mantovani A: Pattern recognition by pentraxins. Adv Exp Med Biol 2009, 653:98-116.

47. Gisladottir B, Gudmundsdottir S, Brown L, Jonsson ZO, Magnadottir B: Isolation of two C-reactive protein homologues from cod (Gadus morhua L.) serum. Fish Shellfish Immunol 2009, 26:210-219.

48. Larkin A, Imperiali B: The expanding horizons of asparagine-linked glycosylation. Biochemistry 2011, 50:4411-4426.

49. Cepeda V, Cofre C, González R, MacKenzie S, Vidal R: Identification of genes involved in immune response of Atlantic salmon (Salmo salar) to IPN virus infection, using expressed sequence tag (EST) analysis. Aquaculture 2011, 318:54-60

50. Alonso MA, Weissman SM: CDNA cloning and sequence of MAL, a hydrophobic protein associated with human T-cell differentiation. Proc Natl Acad Sci U S A 1987, 84:1997-2001.

51. Folch J, Lees M, Sloane-Stanley GH: A simple method for the isolation and purification of total lipides from animal tissues. J Biol Chem 1957, 226:497-509.

52. Christie WW: Lipid analysis. Bridgewater: The Oily Press; 2003.

53. Tacchi L, Bron JE, Taggart JB, Secombes CJ, Bickerdike R, Adler MA, Takle H, Martin SAM: 2011 Multiple tissue transcriptomic responses to Piscirickettsia salmonis in Atlantic salmon (Salmo salar). Physiol Genomics 2011, 43:1241-1254.

54. Krasnov A, Timmerhaus G, Afanasyev S, Jørgensen S-M: Development and assessment of oligonucleotide microarrays for Atlantic salmon (Salmo salar L.). Comp Biochem Physiol Part D Genomics Proteomics 2011, 6:31-38.

55. Brazma A, Hingamp P, Quackenbush J, Sherlock G, Spellman P, Stoeckert C, Aach J, Ansorge W, Ball CA, Causton HC, Gaasterland T, Glenisson P, Holstege FC, Kim IF, Markowitz V, Matese JC, Parkinson H, Robinson A, Sarkans U, Schulze-Kremer S, Stewart J, Taylor R, Vilo J, Vingron M: Minimum information about a microarray experiment (MIAME)-toward standards for microarray data. Nat Genet 2001, 29:365-371.

56. Pfaffl MW, Horgan GW, Dempfle L: Relative expression software tool (REST) for group-wise comparison and statistical analysis of relative expression results in real-time PCR. Nucleic Acids Res 2002, 30:e36.

57. Haugland O, Torgersen J, Syed M, Evensen O: Expression profiles of inflammatory and immune-related genes in Atlantic salmon (Salmo salar L.) at early time post vaccination. Vaccine 2005, 23:5488-5499.

58. Todorcević M, Skugor S, Krasnov A, Ruyter B: Gene expression profiles in Atlantic salmon adipose-derived stromo-vascular fraction during differentiation into adipocytes. BMC Genomics 2010, 11:39.

59. Vandesompele J, De Preter K, Pattyn F, Poppe B, Van Roy N, De Paepe A Speleman F: Accurate normalization of real-time quantitative RT-PCR data by geometric averaging of multiple internal control genes. Genome Biology 2002, 3:34.1-34.11.

\section{doi:10.1186/1471-2164-13-410}

Cite this article as: Morais et al:: Hepatic transcriptome analysis of interfamily variability in flesh $n-3$ long-chain polyunsaturated fatty acid content in Atlantic salmon. BMC Genomics 2012 13:410 